\title{
SPECTRAL ANALYSIS FOR HARMONIZABLE PROCESSES
}

\author{
By KeH-SHIN LII AND MURRAY RosenblatT \\ University of California, Riverside and University of California, San Diego
}

\begin{abstract}
Spectral estimation of nonstationary but harmonizable processes is considered. Given a single realization of the process, periodogram-like and consistent estimators are proposed for spectral mass estimation when the spectral support of the process consists of lines. Such a process can arise in signals of a moving source from array data or multipath signals with Doppler stretch from a single receiver. Such processes also include periodically correlated (or cyclostationary) and almost periodically correlated processes as special cases. We give detailed analysis on aliasing, bias and covariances of various estimators. It is shown that dividing a single long realization of the process into nonoverlapping subsections and then averaging periodogramlike estimates formed from each subsection will not yield meaningful results if one is estimating spectral mass with support on lines with slope not equal to 1 . If the slope of a spectral support line is irrational, then spectral masses do not fold on top of each other in estimation even if the data are equally spaced. Simulation examples are given to illustrate various theoretical results.
\end{abstract}

1. Introduction. Spectral analysis of stationary processes has a long history with interest in both theory and applications. Wide-ranging applications in various practical problems in engineering, economics, science and medicine are well documented [see Yaglom (1987)]. These developments have been closely coupled with the stationarity assumption. In recent years there has been growing interest in the spectral analysis of various specific models of nonstationarity. A class of models of this type that have generated considerable interest are the periodically correlated (or cyclostationary, periodically stationary) or almost periodically correlated processes [Alekseev (1988), Hurd (1989), Gardner (1991), Gerr and Allen (1994a, 1994b), Dandawate and Giannakis (1994) and Leskow and Weron (1992)]. The stationary and almost periodically correlated processes are proper subsets of the class of harmonizable processes. A harmonizable continuous-timeparameter process $X(t), E X(t) \equiv 0$, can be represented as a Fourier-Stieltjes integral [Loève (1963)]

$$
X(t)=\int_{-\infty}^{\infty} e^{i t \lambda} d Z(\lambda)
$$

Received December 1999; revised August 2001.

AMS 2000 subject classifications. Primary, 62M15; secondary, 62G07, 60G12, $62 \mathrm{~F} 12$.

Key words and phrases. Spectral density function, harmonizable processes, estimation, bias, covariance, aliasing, cyclostationary, consistency, Doppler, multipath. 
of a process $Z(\lambda)$ and the covariance function

$$
\begin{aligned}
r_{t, \tau} & =\operatorname{cov}(X(t), X(\tau)) \\
& =\iint_{-\infty}^{\infty} e^{i t \lambda-i \tau \mu} \operatorname{cov}(d Z(\lambda), d Z(\mu)) \\
& =\iint_{-\infty}^{\infty} e^{i t \lambda-i \tau \mu} d H(\lambda, \mu),
\end{aligned}
$$

with the spectral function

$$
\begin{aligned}
& H(\lambda, \mu)=\operatorname{cov}(Z(\lambda), Z(\mu)), \\
& \int_{-\infty}^{\infty} \int|d H(u, v)|<\infty
\end{aligned}
$$

of bounded variation. A readable discussion of the properties of harmonizable processes with many references is given in Yaglom (1987).

In the case of stationary processes, one has a representation of the form (1.1) with $Z(\lambda)$ a process of orthogonal increments, $E(d Z(\lambda) \overline{d Z(\mu)})=\delta_{\lambda, \mu} d F(\lambda)$ and $F$ a bounded nondecreasing function. Major interest focuses on the estimation of the spectral density $f(\lambda)=F^{\prime}(\lambda)$ when $F$ is differentiable. The concept of harmonizability in the sense of Loève is a natural generalization that includes a large class of nonstationary processes. The spectral mass may not concentrate on the diagonal line $\lambda=\mu$ because the process $Z(\lambda)$ in (1.1) may not have orthogonal increments. A natural question concerns what one can estimate consistently about the spectral function $H(\lambda, \mu)$ of a harmonizable process from a single realization $X(t),|t| \leq T$, as $T \rightarrow \infty$.

If the assumption of stationarity is dropped, there are many different nonstationary models that can be considered [see Dahlhaus (1997) for some references and examples]. The basic model treated here differs from that considered in recent papers where nonstationary processes bounded in mean square are considered. Much of the research in these papers [see Dahlhaus (1997), Mallat, Papanicolaou and Zhang (1998) and Neumann and von Sachs (1997)] is based on the concept of a locally stationary process, that is, one that can be approximated locally by a stationary process. If a process has spectral mass with support on lines that are not very close to the diagonal line $\lambda=\mu$, then locally stationary processes are not suitable to model such a process. The approximate methods are based on the asymptotics suggested by the local approximation of the process. Here there is no notion of such an approximation-the fixed given process is analyzed. To be specific, consider a process $X(t)$ with periodic covariance (period $T>0$ ) $\operatorname{cov}(X(t), X(\tau))=R(t, \tau)=\operatorname{cov}(X(t+T), X(\tau+T))$. All discrete time parameter processes of this type are harmonizable but they are not locally stationary if they are periodic but not stationary.

It should be noted that there are continuous-time-parameter periodic processes that are not harmonizable. Gladyshev (1963) gives the example $R(t, \tau)=g(t) g(\tau)$ 
with $g$ continuous periodic with period $T$ but having Fourier coefficients that are not absolutely summable. If the Fourier coefficients are absolutely summable, the process with this covariance function is, of course, harmonizable. Let the number $\tau$ be a translation number of the complex continuous function $f$ belonging to $\varepsilon>0$ if $\sup _{-\infty<x<\infty}|f(x+\tau)-f(x)| \leq \varepsilon$. If for any $\varepsilon>0$ there is an $l(\varepsilon)>0$ such that any interval of length $l(\varepsilon)$ contains a translation number of $f$ belonging to $\varepsilon$, then $f$ is almost periodic in the sense of Bohr $(f \in B)$ [Bohr (1951)]. Any finite trigonometric polynomial $\sum_{n=1}^{m} \alpha_{n} \exp \left(i \lambda_{n} x\right)$ with the $\lambda$ 's real is in $B$. Furthermore, $B$ is an algebra closed under uniform convergence. A process $X(t)$ is said to have an almost periodic covariance function $B(t, \tau)$ if $B(t+s, \tau+s)$ is almost periodic as a function of $s$ for each $t, \tau$. Most processes with almost periodic covariance functions that are not stationary are harmonizable but not locally stationary. The class of processes we consider is qualitatively broader than the processes with almost periodic covariance function and the nonstationary subclass is not locally stationary in general.

In this paper we consider the estimation of spectra of harmonizable processes with spectral mass concentrated on lines on the basis of a time-ordered sequence of observations. Estimation of spectra on lines with slopes not parallel to the diagonal has been of certain interest in signal processing [see Allen and Hobbs (1992)]. Some preliminary results were announced in Lii and Rosenblatt (1998). Further remarks on where such problems arise are given after (2.7), where specific models are discussed. In Section 2 we lay out some basic properties relevant to the estimation problem and motivate the consideration of harmonizable processes with spectral mass concentrated on lines. In Section 3 a periodogram-like estimator is proposed and its bias is examined. The covariance properties of this estimator are given in Section 4. A consistent estimator is considered in Section 5. Remarks are made about the multivariate case. Section 6 has simulated examples and remarks on estimation and computation. Section 7 has most of the proofs. We note that, while the basic form of the estimator is a smoothing of a periodogram-like form, the analysis is quite different from that of the usual periodogram-based estimators. It is shown that averaging periodograms of nonoverlapping subsections of a long section of data will not produce a meaningful result when the slope of the line of the spectral support is not 1 in contrast to what happens in the case of stationary or almost periodically correlated processes. Results obtained include periodically correlated or almost periodically correlated processes as special cases for which much more detailed analysis and results are presented here than in the existing literature. In particular, the effects of aliasing are given. Extensive applications of these processes are well documented in Gardner (1994).

2. Preliminaries. Consider a zero-mean harmonizable process with the representation (1.1). If the process is real, then $r_{t, \tau}=r_{\tau, t}$ and is real. This implies that

$$
d H(u, v)=d H(-v,-u) .
$$


This is symmetry with respect to $u=-v$. Also,

$$
d H(u, v)=\overline{d H(-u,-v)}=\overline{d H(v, u)} .
$$

This is complex conjugate symmetry with respect to the point $(0,0)$ and the line $u=v$. From this we see that information of $H(u, v)$ in the quadrant given by $\{(u, v)|v \geq| u \mid\}$ determines $H(u, v)$ everywhere. If the process $X_{t} \equiv X(t)$ is stationary, the spectral mass is concentrated on the diagonal line $d H(u, v)=$ $\delta_{v-u, 0} \times d F(u)$. If the process $X_{t}$ is periodically or almost periodically correlated, then the spectral mass is concentrated on at most countably many lines which are parallel to the diagonal [Gladyshev (1963) and Dehay and Hurd (1993)]

$$
d H(u, v)=\sum_{j} \delta_{u-v-w_{j}, 0} d F_{j}(u) .
$$

In this paper we assume the continuous-time harmonizable process $X(t)$ has all its spectral mass concentrated on a finite number of lines of the form $u=\alpha v+w$, $\alpha>0$. We will discuss cases in which there is spectral mass on a set of twodimensional positive measure, on a curve which is not a straight line, as well as when $\alpha \leq 0$. We are interested in estimation of the spectral density $f_{\alpha, w}(\mu)$ on a line $\lambda=\alpha \mu+w$ :

$$
\left.d H(\lambda, \mu)\right|_{\lambda=\alpha \mu+w}=\delta_{\lambda-\alpha \mu-w, 0} d F_{\alpha, w}(\mu)=\delta_{\lambda-\alpha \mu-w, 0} f_{\alpha, w}(\mu) d \mu
$$

The special case with $\alpha=1$ was considered in Hurd (1989) and Dandawate and Giannakis (1994). Because of the symmetries in the case of a real-valued process, if $u=a v+b$ is a line of spectral support, then so are $u=a^{-1} v-a^{-1} b, u=a v-b$ and $u=a^{-1} v+a^{-1} b$ with

$$
f_{a, b}(v)=a \bar{f}_{a^{-1},-a^{-1} b}(a v+b)=\bar{f}_{a,-b}(-v)=a f_{a^{-1}, a^{-1} b}(-a v-b) .
$$

Sampling at the integers $n, m$, we have

$$
X(n) \equiv X_{n}=\int_{-\infty}^{\infty} e^{i n \lambda} d Z(\lambda)=\int_{-\pi}^{\pi} e^{i n \lambda} \sum_{k=-\infty}^{\infty} d Z(\lambda+2 \pi k),
$$

with

$$
r_{n, m} \equiv \operatorname{cov}\left(X_{n}, X_{m}\right)=E X_{n} \bar{X}_{m}=\iint_{-\pi}^{\pi} e^{i n \lambda-i m \mu} d \tilde{H}(\lambda, \mu)
$$

and

$$
d \tilde{H}(\lambda, \mu)=\sum_{k, j} d H(\lambda+2 \pi k, \mu+2 \pi j) .
$$

From this it is seen that an aliasing problem (in the sense of spectral mass folding onto $[-\pi, \pi]^{2}$ ) occurs if the harmonizable process is broadband.

It is well known that one generally does not have consistent estimates of spectral mass for a harmonizable process when $\tilde{H}$ is absolutely continuous with a 
spectral density $\tilde{h}, d \tilde{H}(\lambda, \mu)=\tilde{h}(\lambda, \mu) d \lambda d \mu$ with $\tilde{h}(\lambda, \mu) \neq 0$ on a set of twodimensional Lebesgue measure, if the sample is $X_{-n}, \ldots, X_{n}$, letting $n \rightarrow \infty$. The simplest example is given by $X_{0} \sim N(0,1)$ with $X_{k}=0$ for $k \neq 0$, where $d H(\lambda, \mu)=C d \lambda d \mu$ for some constant $C$. The most one could possibly hope for is to estimate spectral mass whose support has two-dimensional Lebesgue measure 0. The simplest such support is a finite collection of curves and, in particular, lines. This motivates the consideration of estimation of spectral mass concentrated on lines given a single realization.

A simple example of a harmonizable process with spectral mass on lines is given by

$$
X_{t}=Y_{t}+\sum_{s=1}^{k} \beta_{s} Y_{\alpha_{s} t},
$$

with $Y_{t}$ stationary and $\beta_{s}$ and $\alpha_{s}$ real and positive numbers, respectively. In such case the spectral support lines are $\lambda=\mu, \lambda=\alpha_{s} \mu, \lambda=\alpha_{s}^{-1} \mu$ and $\lambda=\alpha_{s} \alpha_{s^{\prime}}^{-1} \mu$ for all $s, s^{\prime}=1, \ldots, k$. In Section 6 we give a slightly more complicated example where it is shown that the spectral support lines have the given form.

A small variant of this model is given by $X_{t}=\sum_{s=1}^{k} \beta_{s} Y_{\alpha_{s}\left(t-\tau_{s}\right)}$. Such processes can be encountered in contexts when signals are of a multipath character and contain time delays $\tau_{s}$ and Doppler stretches $\alpha_{s}$ due to different propagation speed along different paths for a single receiver. For a multiple-receiver example, consider an acoustic signal that emanates from a moving point source and propagates through an acoustic medium, before being received at two spatially separated sensors. If, for instance, the source is in motion while the sensors are stationary, then the actual waveform observed at the two sensor locations can be modeled by [Ferguson (1999), page 261].

$$
\begin{aligned}
& x_{1}(t)=s\left(\beta_{1} t\right)+v_{1}(t), \\
& x_{2}(t)=\sigma s\left(\beta_{2}(t-d)\right)+v_{2}(t),
\end{aligned}
$$

where $v_{1}(t)$ and $v_{2}(t)$ represent additive noise observed in the absence of signal, $d$ is the time delay, $\sigma$ is the relative attenuation and $\beta_{1}, \beta_{2}$ are the respective receiver time scales that are introduced to account for the differential Doppler effect. In the present case, the relative motion between the source and the sensors results in the radial velocity component of the source at any given time being different for each receiver. An example of aircraft transits overhead illustrating this model is given in Ferguson (1999), page 261. Alternatively, the received waveforms can be modeled by

$$
\begin{aligned}
& x(t)=s(t)+n_{x}(t), \\
& y(t)=\sigma_{1} s(\beta(t-\tau))+n_{y}(t),
\end{aligned}
$$

where $\beta=\beta_{1} / \beta_{2}$ [Ferguson (1999), (16)]. It is also demonstrated in Ferguson that if the Doppler effect is ignored then the estimated delay will not be correct. 
Equation (16) of Ferguson (1999) is a version of a special case of the model we dealt with (see the remarks at the end of Section 5 on multivariate harmonizable processes). Additional examples can be found in Jin, Wong and Luo (1995), page 904, for a wideband communication example and in Munk, Worcester and Wunsch (1995), page 204, for one in ocean acoustic tomography. A related example can be found in Chiu (1986) for seismic applications.

Throughout the paper we shall assume that $X(t)$ is a continuous-time-parameter harmonizable process with:

ASSUMPTION 1. All spectral support is on a finite number of lines given by $\mathcal{L}=\left\{\left(a_{i}, b_{i}\right) \mid u=a_{i} v+b_{i}, i=1, \ldots, K\right\}$ with positive slopes $a_{i}>0$.

Assumption 2. The spectral mass on the line $u=a v+b$ is given by a continuously differentiable spectral density $f_{a, b}(v),(a, b) \in \mathcal{L}$. The spectral densities $f_{a, b}(v)$ and their first derivatives are continuous and bounded in absolute value by a function $g(v)$ that is a monotonic decreasing function of $|v|$ that decreases to 0 as $|v| \rightarrow \infty$ and that is integrable as a function of $v$.

The following simple remark indicates how a harmonizable process with line spectra may differ from a stationary or almost periodically correlated process in terms of aliasing. The aliasing then can have a more complicated character. We say that a line $u=a v+b$ is a line of spectral support if the spectral density $f_{a, b}(v)$ on the line is nonzero on a set of positive one-dimensional Lebesgue measure.

PROPOSITION 2.1. Let $X(t)$ be a continuous-time-parameter process continuous in mean square satisfying Assumptions 1 and 2. The discretely observed process $X_{n}$ then has a countably infinite number of lines of support in $(-\pi, \pi]^{2}$ if and only if one of the lines of spectral support of $X(t)$ has irrational slope $a$ and its spectral density is nonzero at an infinite number of points $v_{j}$, with $\left|v_{j}\right| \rightarrow \infty$. Furthermore, if there is a line of spectral support with irrational slope with spectral density nonzero at all $v,|v|>s$ for some $s>0$, then the discretely observed process has a countably dense set of lines of support in $(-\pi, \pi]^{2}$.

Before we prove the proposition, we set the following convention which will be followed in the paper. When we refer to $w=z \bmod 2 \pi$, it is understood that $-\pi<w \leq \pi$. Let $\{u\}$ be the integer $l$ such that $-1 / 2<u-l \leq 1 / 2$. Our version of $z \bmod 2 \pi$ is then $(z \bmod 2 \pi)=z-\{z /(2 \pi)\} 2 \pi$.

Proof of Proposition 2.1. The proposition follows from the following simple remarks. Let $u=a v+b$ be a line with nonzero spectral density for the continuous-time process. The aliased line segments of this line, in the discretely sampled case, in $(-\pi, \pi]^{2}$ all have slope $a$. Let us look at the intersections of these line segments with $v=v_{0} \in(-k \pi, k \pi],-\pi<u \leq \pi$, for integer $k$. The points 
$\left(a\left(v_{0}+2 \pi n\right)+b, v_{0}+2 \pi n\right), n$ integer, on $u=a v+b$ are mapped onto the points $\left(\left(2 \pi n a+a v_{0}+b\right) \bmod 2 \pi, v_{0}\right)$ of $v=v_{0},-\pi<u \leq \pi$. If $a$ is rational there are at most a finite number of values $\left(2 \pi n a+a v_{0}+b\right) \bmod 2 \pi$ for $n$ integer. Notice that if $a=1$ there are at most two such aliased line segments. If $a$ is irrational there are an infinite number of values $\left(2 \pi n a+a v_{0}+b\right) \bmod 2 \pi$ for $n$ integer and they are dense on $(-k \pi, k \pi]$. Since $v_{0}$ is an arbitrary point in $(-k \pi, k \pi]$, the aliased line segments in $(-\pi, \pi]^{2}$ are dense. Now the proposition follows using Assumptions 1 and 2.

We note that the previous argument also implies that spectral mass with its support on a line with irrational slope never folds back on its own aliased line segments. Equally spaced sampling of a continuous-time process does produce aliasing. In the stationary case the "Nyquist" frequency is the frequency such that spectral mass beyond that frequency folds back on spectral mass of a lower frequency. In the harmonizable case aliasing does not produce a Nyquist frequency in the conventional sense for a spectral density whose spectral line support has irrational slope. Spectral masses of two different frequencies do not overlap due to aliasing in the case of a spectral density with support line having irrational slope. In this sense there is no overlap "aliasing problem" for spectral density estimation if the support line has irrational slope. The effect of aliasing on estimation in almost periodically correlated processes has not appeared in the literature and is given as a special case of our analysis in Sections 3-5.

3. A periodogram and its bias. In the classical case of a stationary process, an estimate proposed by Daniell (1946) was obtained by dividing a sequence of observations $X_{0}, \ldots, X_{N-1}$ into $m$ disjoint sections of length $n, N=$ $m n$, computing a periodogram for each section and averaging the resulting periodograms. On letting $m, n \rightarrow \infty$ as $N \rightarrow \infty$, a consistent estimate is obtained under appropriate conditions. If a harmonizable process has mass on a line of slope $a \neq 1$, one object of our discussion is to show that a Daniell-like estimate cannot be used to estimate the spectral mass on that line.

To show the effect relative to a Daniell-like estimate in sampling from the continuous-time harmonizable process $X(t),-\infty<t<\infty$, let us consider observations $X_{t+l J}$ with $t=0, \ldots, n-1$ with $J, n \geq 0$ integers and $l$ an index value. Let

$$
F_{l, n}(\lambda)=\sum_{t=0}^{n-1} X_{t+l J} e^{-i(t+l J) \lambda},
$$

with corresponding periodogram

$$
I_{l, n}(\lambda, \mu)=\frac{1}{2 \pi n} F_{l, n}(\lambda) \overline{F_{l, n}(\mu)} .
$$

Notice that, for $J=n, F_{l, n}(\lambda)$ uses nonoverlapping subsections of $X(t)$ for different integers $l$. When $J<n$, we have overlapping sections [Zurbenko (1986), 
page 214]. Given a sample $X_{0}, \ldots, X_{N-1}, N=m n$, a Daniell-like estimate is, when $J=n$, the average of periodograms of nonoverlapping subsections of the data,

$$
D_{m, n}(\lambda, \mu)=\frac{1}{m} \sum_{l=0}^{m-1} I_{l, n}(\lambda, \mu) .
$$

Another concern is to show the effect of aliasing that is the consequence of discretely sampling a continuous-time-parameter process. In the case of a stationary process, one tries to correct the aliasing by either narrowband filtering or discretely sampling more frequently. Similar methods can be used for the processes we deal with, but it should be noted that the effects of aliasing are more complicated than they are for a stationary process.

Given $(\lambda, \mu) \in(-\pi, \pi]^{2}$, let $(\alpha, w)$ be a fixed pair with $\alpha>0$ such that $\lambda=\alpha \mu+w$. Then set

$$
\begin{aligned}
y & \equiv y(k ; a, b ; \lambda, \mu) \\
& \equiv((2 \pi k a+(a-\alpha) \mu+b-w) \bmod 2 \pi) \\
& =((2 \pi k a+a \mu+b-\lambda) \bmod 2 \pi)
\end{aligned}
$$

for $(a, b) \in \mathcal{L}$ with $k$ an integer. The meaning of some aspects of $y$ will be remarked on later. One can then obtain the following theorem.

THEOREM 3.1. Assume Assumptions 1 and 2 hold. Then

$$
\begin{aligned}
E I_{l, n}(\lambda, \mu)= & \sum_{(a, b) \in \mathcal{L}} \sum_{|y(k ; a, b ; \lambda, \mu)| \leq \nu} e^{i l J y(k ; a, b ; \lambda, \mu)} \\
& \times f_{a, b}(\mu+2 \pi k) G(n, l, J, y(k ; a, b ; \lambda, \mu)) \\
& +O\left(\frac{\log n}{n}\right)
\end{aligned}
$$

uniformly in $(\lambda, \mu)$ with a fixed $v \in(0, \pi]$ and

$$
\begin{aligned}
G & =G(n, l, J, y(k ; a, b ; \lambda, \mu)) \\
& =\frac{1}{a} \int_{-\infty}^{\infty} 1_{[0, a]}(t) 1_{[-l J(a-1) / n, 1-l J(a-1) / n]}(t) e^{i n y t / a} d t \\
& =\left\{\begin{array}{c}
\frac{1}{\text { iny }}\left[\exp \left(\frac{\text { iny }}{a}\left[\min \left(a, 1-\frac{l J(a-1)}{n}\right)\right]\right)\right. \\
\left.-\exp \left(\frac{i n y}{a} \max \left(0,-\frac{l J(a-1)}{n}\right)\right)\right], \\
\text { if }(0, a) \cap\left(-\frac{l J(a-1)}{n}, 1-\frac{l J(a-1)}{n}\right) \neq \varnothing, \\
0, \quad \text { otherwise, }
\end{array}\right.
\end{aligned}
$$

where $\varnothing$ is the empty set. 
We note that for an error term like that in (3.5) we need $v$ fixed and cannot let $v \downarrow 0$.

The theorem gives a detailed estimate for the expectation of this type of periodogram. It is worthwhile making a few remarks about the theorem. First, notice that if $(a, b) \in \mathcal{L}$ and $y(k ; a, b ; \lambda, \mu) \neq 0$ for a fixed $k$ the contribution to the expectation from that term in the double sum on the right-hand side of (3.5) [see (3.6)] is $O(1 / n)$. For an $(a, b) \in \mathcal{L}$, the condition

$$
y=((2 \pi k a-\lambda+(a \mu+b)) \bmod 2 \pi)=0
$$

for some integer $k$ means that the line of spectral support $u=a v+b$ or an aliased version of it passes through the point $(\lambda, \mu)$. From (3.4) we also see that $y=0$ implies $2 \pi k a+a \mu+b-(\alpha \mu+w)=0 \bmod 2 \pi$. Notice that if $(\alpha, w) \in \mathcal{L}$ with $(a, b)=(\alpha, w)$ and $k=0$ then $y=0$ is satisfied. This means that if $(\lambda, \mu)$ is a point on a spectral support line then there is a contribution to (3.5) in the summation. If $(\alpha, w) \notin \mathcal{L}$ then $y=0$ in (3.4) implies there exists $(a, b) \in \mathcal{L}$ such that at least one of its aliased lines (i.e., at least one $k$ ) passes through the point $(\lambda, \mu)$. So for a given point $(\lambda, \mu)$ if it is not on any spectral support lines or their aliased versions then $E I_{l, n}(\lambda, \mu)=O(\log n / n)+G(M)$, where

$$
G(M)=\sum_{(a, b) \in \mathcal{L}|y| \leq \nu} \sum_{\mid \text {and }|k|>M} e^{i l J y} f_{a, b}(\mu+2 \pi k) G(n, l, J, y)
$$

for any fixed integer $M$ from (3.4) and (3.5). However, $|G(M)| \rightarrow 0$ as $M \rightarrow \infty$ by Assumptions 1 and 2 . The rate at which $G(M) \rightarrow 0$ depends on the rate of $g(v) \rightarrow 0$ in Assumption 2. As a result we have:

COROLlaRY 3.1. Under Assumptions 1 and 2, if $(\lambda, \mu) \in[-\pi, \pi]^{2}$ is not on any lines given by $(a, b) \in \mathcal{L}$ or their aliased lines, then $E I_{l, n}(\lambda, \mu) \rightarrow 0$ as $n \rightarrow \infty$.

For the term with $a=1$, we have

$$
G=\frac{1}{\text { iny }}\left[e^{i n y}-1\right] .
$$

Then, if $y \neq 0, y=((a \mu+b-\lambda) \bmod 2 \pi)$ is fixed and independent of $k$ and $G=O(1 / n)$ whatever the values of $l$ and $J$. If $a \neq 1$, then $G \rightarrow 0$ if $l J / n \rightarrow \infty$ as $n \rightarrow \infty$. Also, if $y(k ; a, b ; \lambda, \mu)=0$, then, for $a>0$,

$$
G=\frac{1}{a}\|[0, a] \cap[-l J(a-1) / n, 1-l J(a-1) / n]\|,
$$

with $\|\cdot\|$ denoting length. Notice that, if $y=0$, then $G \neq 0$ if and only if either

$$
a \leq 1 \text { and }-\frac{1}{1-a}<\frac{l J}{n}<\frac{a}{1-a}
$$


or

$$
a \geq 1 \quad \text { and } \quad-\frac{a}{a-1}<\frac{l J}{n}<\frac{1}{a-1} .
$$

To guarantee that $G \neq 0$ for arbitrary $a>0$, we need $-1 \leq l J / n \leq 0$. Also notice that, from (3.7),

$$
G=\frac{1}{a} \min (a, 1)
$$

if (i) $a=1$ or $l=0$ for any $J$ or (ii) $l \in[-1,0]$ and $J=n$. This suggests that, for $J=n$ and $y=0$, any $l \in[-1,0]$ can be used in (3.1) and in these cases $G$ terms in (3.5) have a simple form. However, for computational purposes, the following three forms of a finite Fourier transform, which correspond to the cases when $J=n$ and $l=0,-1$ and $-\frac{1}{2}$, respectively, in (3.1), can be conveniently used, for any $a>0$,

$$
\begin{aligned}
& \text { (a) } F_{n}(\lambda)=\sum_{t=0}^{n} X_{t} e^{-i t \lambda}, \\
& \text { (b) } F_{n}(\lambda)=\sum_{t=-n}^{0} X_{t} e^{-i t \lambda}, \\
& \text { (c) } F_{n}(\lambda)=\sum_{t=-n / 2}^{n / 2} X_{t} e^{i t \lambda},
\end{aligned}
$$

with a corresponding periodogram

$$
I_{n}(\lambda, \mu)=\frac{1}{2 \pi(n+1)} F_{n}(\lambda) \overline{F_{n}(\mu)}
$$

We will use the form (c) given by (3.8) later and in such cases we take $n$ to be even.

COROLlary 3.2. Assume Assumptions 1 and 2 hold and $J=n$ with $l \in$ $[-1,0]$. Then, as $n \rightarrow \infty$,

$$
E I_{l, n}(\lambda, \mu) \rightarrow \sum_{(a, b) \in \mathcal{L}} \sum_{y(k ; a, b ; \lambda, \mu)=0} f_{a, b}(\mu+2 \pi k) \frac{1}{a} \min (a, 1) .
$$

Notice that if there is no aliasing the right-hand side of (3.10) becomes

$$
\sum_{(a, b) \in \mathcal{L}} \sum_{y(k ; a, b ; \lambda, \mu)=0} f_{a, b}(\mu) \frac{1}{a} \min (a, 1) .
$$


Since we assume only a finite number of lines of spectral support, there are at most a finite number of points of intersection of these lines. Aside from these points, the mean value tends to

$$
f_{\alpha, w}(\mu) \frac{1}{\alpha} \min (\alpha, 1)
$$

if $\lambda=\alpha \mu+w$ is a line of spectral support. In the case of a Daniell-like estimate (3.3) (note $J=n$ ), where $l$ takes on integer index values, if $a \neq 1$ then, except for a fixed number of terms, $|l(a-1)|>1$ and so the corresponding $G$ [see (3.7)] value is 0 . Alternatively, we saw earlier that, to guarantee $G \neq 0$ for arbitrary $a>0$, we needed $l \in[-1,0]$ when $J=n$. Consequently, the mean of the Daniell estimate (3.3) which averages over periodograms of nonoverlapping subsections of the data will generally not tend to $f_{\alpha, w}(\cdot)$ as $m, n \rightarrow \infty$ even when $\alpha<1$.

If a process is almost periodically correlated, the spectral mass is located on lines of slope 1 and $y(k ; 1, b ; \mu+w, \mu)=(b-w) \bmod 2 \pi$. The following corollary holds.

COROLLARY 3.3. If the process is almost periodically correlated, under Assumptions 1 and 2 one has

$$
E I_{l, n}(\lambda, \mu)=\sum_{(1, b) \in \mathcal{L}} \sum_{|y(k ; 1, b ; \mu+w, \mu)| \leq v} e^{i l J y(k ; 1, b ; \mu+w, \mu)}
$$

$$
\begin{aligned}
& \times f_{1, b}(\mu+2 \pi k) \frac{1}{i n y}\left[e^{i n y}-1\right] \\
+ & O\left(\frac{\log n}{n}\right) .
\end{aligned}
$$

As $n \rightarrow \infty$, the expected value tends to

$$
\sum_{(1, b) \in \mathcal{L}} \sum_{y(k ; 1, b ; \mu+w, \mu)=0} f_{1, b}(\mu+2 \pi k) .
$$

Notice that $y(k ; 1, b ; \mu+w, \mu)=(b-w) \bmod 2 \pi$ from (3.4).

From the previous discussions we see that the expectations of the periodogram from an equally spaced discrete sample [see (3.1)] asymptotically converge to the "aliased" version of the spectral density function on the support lines. This is analogous to the stationary case where the expectation of the periodogram converges asymptotically to the aliased version of the spectral density function. The difference is that the aliasing is more complicated for the harmonizable processes than for the stationary processes. Bias and consistency properties of an estimator based on discrete samples are properties relative to these aliased spectral densities. We also note here that when the spectral support line has slope $a<0$, previous and subsequent discussions and results still hold if we use the 
symmetrized version (c) in (3.8) with very minor modifications in the derivations. We have degenerate cases when the slope of the spectral support line is $a=0$ which is symmetric to the line with slope $a^{-1}=\infty$. It is also plausible, based on the discussions in this section, that if the spectral mass is concentrated on a smooth curve, similar results on the expectations and covariances should hold. These details are not pursued here.

4. Periodogram covariance. Our objective is to now give the asymptotic behavior of the covariance of the periodogram. Given fixed $(\lambda, \mu),\left(\lambda^{\prime}, \mu^{\prime}\right)$, assume that the fixed pair $(\alpha, w),\left(\alpha^{\prime}, w^{\prime}\right)$ with $\alpha>0, \alpha^{\prime}>0$ is such that $\lambda=\alpha \mu+w$ and $\lambda^{\prime}=\alpha^{\prime} \mu^{\prime}+w^{\prime}$. Let $(a, b),\left(a^{\prime}, b^{\prime}\right) \in \mathcal{L}$. To state the result in a convenient form, the following expressions are introduced:

$$
\begin{aligned}
& y(1)=\left(y\left(k ; a, b ; \lambda, \lambda^{\prime}\right)\right)=\left(\left(2 \pi k a+a \lambda^{\prime}+b-\lambda\right) \bmod 2 \pi\right), \\
& y(2)=\left(y\left(k^{\prime} ; a^{\prime}, b^{\prime} ;-\mu,-\mu^{\prime}\right)\right)=\left(\left(2 \pi k^{\prime} a^{\prime}-a^{\prime} \mu^{\prime}+\mu+b^{\prime}\right) \bmod 2 \pi\right), \\
& y(3)=\left(y\left(k ; a, b ; \lambda,-\mu^{\prime}\right)\right)=\left(\left(2 \pi k a-a \mu^{\prime}+b-\lambda\right) \bmod 2 \pi\right), \\
& y(4)=\left(y\left(k^{\prime} ; a^{\prime}, b^{\prime} ;-\mu, \lambda^{\prime}\right)\right)=\left(\left(2 \pi k^{\prime} a^{\prime}+a^{\prime} \lambda^{\prime}+\mu+b^{\prime}\right) \bmod 2 \pi\right) .
\end{aligned}
$$

This additional notation is introduced so that formulas later on do not become too cluttered. Notice that in (4.1)-(4.4) two lines $\lambda=\alpha \mu+w, \lambda^{\prime}=\alpha^{\prime} \mu^{\prime}+w^{\prime}$ are dealt with. The notation in this case is introduced to take care of the covariance computation. In formula (3.4) the bias of a point on a single line was involved.

The covariance properties of the periodogram are given in the following theorem.

THEOREM 4.1. Let $X(t)$ be a normal harmonizable process satisfying Assumptions 1 and 2. Then

$$
\begin{aligned}
\operatorname{cov}( & \left.I_{l, n}(\lambda, \mu), I_{l, n}\left(\lambda^{\prime}, \mu^{\prime}\right)\right) \\
= & {\left[\sum_{(a, b) \in \mathcal{L}} \sum_{|y(1)| \leq v} f_{a, b}\left(\lambda^{\prime}+2 k \pi\right) e^{i l J y(1)} G(n, l, J, y(1))+O\left(\frac{\log n}{n}\right)\right] } \\
(4.5) \times & {\left[\sum_{\left(a^{\prime}, b^{\prime}\right) \in \mathcal{L}} \sum_{|y(2)| \leq \nu} f_{a^{\prime}, b^{\prime}}\left(-\mu^{\prime}+2 k^{\prime} \pi\right) e^{i l J(2)} G(n, l, J, y(2))+O\left(\frac{\log n}{n}\right)\right] } \\
& +\left[\sum_{(a, b) \in \mathcal{L}} \sum_{|y(3)| \leq \nu} f_{a, b}\left(-\mu^{\prime}+2 \pi k\right) e^{i l J y(3)} G(n, l, J, y(3))+O\left(\frac{\log n}{n}\right)\right] \\
& \times\left[\sum_{\left(a^{\prime}, b^{\prime}\right) \in \mathcal{L}} \sum_{|y(4)| \leq \nu} f_{a^{\prime}, b^{\prime}}\left(\lambda^{\prime}+2 \pi k^{\prime}\right) e^{i l J y(4)} G(n, l, J, y(4))+O\left(\frac{\log n}{n}\right)\right],
\end{aligned}
$$

with $-\pi<\lambda, \lambda^{\prime}, \mu, \mu^{\prime} \leq \pi, v$ given in Theorem 3.1 and $G(\cdot)$ as given in (3.6). 
Theorem 4.1 will be useful in deriving the covariance properties of locally smoothed estimates of the spectral density on lines of spectral support.

COROLLARY 4.1. The result of Theorem 4.1 holds for non-Gaussian harmonizable processes with finite fourth-order moments if the fourth-order cumulants satisfy $\frac{1}{n} \sup _{t} \sum_{\tau, t^{\prime}, \tau^{\prime}}\left|\operatorname{cum}\left(X_{t}, X_{\tau}, X_{t^{\prime}}, X_{\tau^{\prime}}\right)\right| \rightarrow 0$ as $n \rightarrow \infty$. This condition is satisfied if $\sup _{t} \sum_{\tau, t^{\prime}, \tau^{\prime}}\left|\operatorname{cum}\left(X_{t}, X_{\tau}, X_{t^{\prime}}, X_{\tau^{\prime}}\right)\right|<\infty$.

5. Consistent estimates. The estimates that we consider are smoothed versions of the periodogram and for that reason we introduce the following additional assumption.

ASSUMPTION 3. Let $K(\eta)$ be a continuous nonnegative symmetric weight function of finite support with $\int K(x) d x=1$. Also, $K_{n}(\eta)=b_{n}^{-1} K\left(b_{n}^{-1} \eta\right)$, where $b_{n} \downarrow 0$ and $n b_{n} \rightarrow \infty$ as $n \rightarrow \infty$.

The periodogram has the doubly periodic property

$$
I_{n}(\lambda+2 \pi k, \mu+2 \pi j)=I_{n}(\lambda, \mu)
$$

for all $\lambda, \mu$ and all integers $k, j$. This suggests that

$$
\hat{f}_{\alpha, w}(\eta)=\int_{-\pi^{+}}^{\pi^{+}} I_{n}(\alpha \mu+w, \mu) K_{n}(\mu-\eta) d \mu
$$

where $\pi^{+}=\pi+\delta(\delta>0)$ is a plausible estimate of $f_{\alpha, w}(\eta)$ with $-\pi \leq \eta, \alpha \eta+$ $w \leq \pi$.

For convenience in exposition, form (c) of (3.8) of the finite Fourier transform will be used in (3.9). Let us then note that we have, from (3.5) and (4.5), Theorem 5.1.

THEOREM 5.1. Under Assumptions 1 and 2 and $I_{n}(\lambda, \mu)$ given by (3.9) and (3.8)(c), using (3.4),

$$
\begin{aligned}
E I_{n}(\lambda, \mu)= & \sum_{(a, b) \in \mathcal{L}} \sum_{|y| \leq v} f_{a, b}(\mu+2 \pi k) \sin \left(\frac{n+1}{2 a} y \min (a, 1)\right) /\left(\frac{n+1}{2} y\right) \\
& +O\left(\frac{\log n}{n}\right)
\end{aligned}
$$


uniformly in $(\lambda, \mu)$ with $y$ given in (3.4) with the sum over $k$ through the expression $y$ and

$$
\operatorname{cov}\left(I_{n}(\alpha \mu+w, \mu), I_{n}\left(\alpha^{\prime} \mu^{\prime}+w^{\prime}, \mu^{\prime}\right)\right)
$$

$$
\begin{aligned}
= & {\left[\sum_{(a, b) \in \mathcal{L}} \sum_{|y(1)| \leq \nu} f_{a, b}\left(\lambda^{\prime}+2 k \pi\right) \operatorname{sinc}(\alpha, y(1))+O\left(\frac{\log n}{n}\right)\right] } \\
& \times\left[\sum_{\left(a^{\prime}, b^{\prime}\right) \in \mathcal{L}} \sum_{|y(2)| \leq \nu} f_{a^{\prime}, b^{\prime}}\left(-\mu^{\prime}+2 k^{\prime} \pi\right) \operatorname{sinc}\left(\alpha^{\prime}, y(2)\right)+O\left(\frac{\log n}{n}\right)\right] \\
& +\left[\sum_{(a, b) \in \mathcal{L}} \sum_{|y(3)| \leq \nu} f_{a, b}\left(-\mu^{\prime}+2 \pi k\right) \operatorname{sinc}(\alpha, y(3))+O\left(\frac{\log n}{n}\right)\right] \\
& \times\left[\sum_{\left(a^{\prime}, b^{\prime}\right) \in \mathscr{L}} \sum_{|y(4)| \leq \nu} f_{a^{\prime}, b^{\prime}}\left(\lambda^{\prime}+2 \pi k^{\prime}\right) \operatorname{sinc}\left(\alpha^{\prime}, y(4)\right)+O\left(\frac{\log n}{n}\right)\right]
\end{aligned}
$$

for $-\pi<\lambda, \lambda^{\prime}, \mu, \mu^{\prime} \leq \pi$, where $y(1), \ldots, y(4)$ are given in (4.1)-(4.4) and

$$
\begin{aligned}
\operatorname{sinc}(a, y) & =\sin \left(\frac{n+1}{2 a} y \min (a, 1)\right) /\left(\frac{n+1}{2} y\right) \\
& =\operatorname{sinc}\left(\frac{n+1}{2} y l(a)\right) l(a),
\end{aligned}
$$

with

$$
l(a)=\frac{\min (a, 1)}{a}, \quad \operatorname{sinc}(x)=\frac{\sin x}{x} .
$$

Then, by (5.2), (5.3) and (5.5),

$$
\begin{aligned}
E \hat{f}_{\alpha, w}(\eta)= & \sum_{(a, b) \in \mathcal{L}} \sum_{|y| \leq \nu} \int f_{a, b}(\mu+2 \pi k) \operatorname{sinc}(a, y) K_{n}(\mu-\eta) d \mu \\
& +O\left(\frac{\log n}{n}\right),
\end{aligned}
$$

where $y$ is given in (3.4). We then have the following result.

THEOREM 5.2. Under Assumptions 1-3 when $b_{n} \downarrow 0, n b_{n} \rightarrow \infty$ with $\xi=$ $\alpha \eta+w$,

$$
E \hat{f}_{\alpha, w}(\eta)=o\left(b_{n}\right)+O\left(\frac{\log n}{n b_{n}}\right)
$$

if $\alpha$ is not a slope of a spectrum support line. However,

$$
\begin{aligned}
E \hat{f}_{\alpha, w}(\eta)= & l(\alpha) \sum_{k,(\alpha, b) \in \mathcal{L}} f_{\alpha, b}(\eta+2 \pi k) \frac{\sin \left(((n+1) / 2) l(\alpha) y^{\prime}\right)}{((n+1) / 2) y^{\prime} l(\alpha)} \\
& +o\left(b_{n}\right)+o\left(\frac{\log n}{n}\right)
\end{aligned}
$$

if $\alpha$ is a slope of a spectrum support line with $y^{\prime}=((2 \pi k \alpha+b-w) \bmod 2 \pi)$. 
Equation (5.7) says, asymptotically, if $(\xi, \eta)$ is not on a spectral support line or, alternatively, if it is on a spectral support line but the smoothing in the estimate is not on a spectral support line, then $E \hat{f}_{\alpha, w}(\eta) \rightarrow 0$. If $(\xi, \eta)$ is on a spectral support line and the smoothing is on the spectral support line given by $(\alpha, w)$ which passes through $(\xi, \eta)$ (there could be more than one support line passing through the same point), then $E \hat{f}_{\alpha, w}(\eta)$ converges to $l(\alpha)$ times the sum of all spectral masses on spectral support lines which have the same slope $\alpha$ and which are aliased onto $(\xi, \eta)$ through the condition $y^{\prime}=0$.

In the almost periodically correlated case, a simpler estimate leads to the following proposition.

PROPOSITION 5.1. If the process $X(t)$ is almost periodically correlated, then under Assumptions 1-3, $b_{n} \downarrow 0, n b_{n} \rightarrow \infty$,

$$
\begin{aligned}
E \hat{f}_{1, w}(\eta)=\sum_{(1, b) \in \mathcal{L}} \sum_{k} \frac{\sin ((n+1) / 2)((b-w) \bmod 2 \pi))}{((n+1) / 2)((b-w) \bmod 2 \pi)} \\
\times \int f_{1, b}(\mu+2 \pi k) K_{n}(\mu-\eta) d \mu+O\left(\frac{\log n}{n}\right) .
\end{aligned}
$$

If the functions $f_{1, b}$ are continuously differentiable up to second order, then

$$
\begin{aligned}
& \int f_{1, b}(\mu+2 \pi k) K_{n}(\mu-\eta) d \mu \\
& \quad=f_{1, b}(\eta+2 \pi k)+\frac{b_{n}^{2}}{2} f_{1, b}^{\prime \prime}(\eta+2 \pi k) \int z^{2} K(z) d z+o\left(b_{n}^{2}\right)
\end{aligned}
$$

and

$$
\begin{aligned}
E \hat{f}_{1, w}(\eta)= & \sum_{(1, b) \in \mathcal{L}} \sum_{k} \operatorname{sinc}\left(\frac{n+1}{2}((b-w) \bmod 2 \pi)\right) f_{1, b}(\eta+2 \pi k) \\
& +\frac{b_{n}^{2}}{2} \int z^{2} K(z) d z \sum_{(1, b) \in \mathcal{L}} \sum_{k} \operatorname{sinc}\left(\frac{n+1}{2}((b-w) \bmod 2 \pi)\right) \\
& \times f_{1, b}^{\prime \prime}(\eta+2 \pi k) \\
& +o\left(b_{n}^{2}\right)+o\left(\frac{\log n}{n}\right) .
\end{aligned}
$$

We note that if $((b-w) \bmod 2 \pi) \neq 0$, then the first term on the right-hand side of the last equation is $O(1 / n)$. If $((b-w) \bmod 2 \pi)=0$, then the sinc function is 1 . We now consider the asymptotic behavior of the covariance of spectral estimates. 
THEOREM 5.3. Let $(\alpha, w),\left(\alpha^{\prime}, w^{\prime}\right)$ be fixed with $\alpha>0, \alpha^{\prime}>0$. Also, let $(a, b),\left(a^{\prime}, b^{\prime}\right)$ range over the finite set $\mathcal{L}$. If $-\pi \leq \xi, \xi^{\prime}, \eta, \eta^{\prime} \leq \pi$ with $\xi=$ $\alpha \eta+w, \xi^{\prime}=\alpha^{\prime} \eta^{\prime}+w^{\prime}$, then

$$
\begin{aligned}
\operatorname{cov}\left(\hat{f}_{\alpha, w}(\eta), \hat{f}_{\alpha^{\prime}, w^{\prime}}\left(\eta^{\prime}\right)\right) & \\
=\frac{2 \pi}{(n+1) b_{n}}\left\{\sum_{\substack{k, k^{\prime}, a, a^{\prime} \\
b, b^{\prime}}}[\right. & \delta_{a \alpha^{\prime}-a^{\prime} \alpha, 0} \delta_{\left(y\left(k ; a, b ; \xi, \xi^{\prime}\right)\right), 0} \delta_{\left(y\left(k^{\prime} ; a^{\prime}, b^{\prime} ;-\eta,-\eta^{\prime}\right)\right), 0} \\
& \times f_{a, b}\left(a^{\prime} \eta^{\prime}+w^{\prime}+2 \pi k\right) f_{a^{\prime}, b^{\prime}}\left(-\eta^{\prime}+2 \pi k^{\prime}\right) \\
& \times \int K\left(a^{\prime} x\right) K(x) d x \min \left(\alpha^{\prime} \frac{\min (a, 1)}{\min \left(a^{\prime}, 1\right)}, 1\right) \frac{\min \left(a^{\prime}, 1\right)}{a \alpha^{\prime}} \\
+ & \delta_{a-a^{\prime} \alpha^{\prime} \alpha, 0} \delta_{\left(y\left(k^{\prime} ; a^{\prime}, b^{\prime} ;-\eta, \xi^{\prime}\right)\right), 0} \delta_{\left(y\left(k ; a, b ; \xi,-\eta^{\prime}\right)\right), 0} \\
& \times f_{a, b}\left(-\eta^{\prime}+2 \pi k\right) f_{a^{\prime}, b^{\prime}}\left(a^{\prime} \eta^{\prime}+w^{\prime}+2 \pi k^{\prime}\right) \\
& \times \int K\left(-a^{\prime} \alpha^{\prime} x\right) K(x) d x \min \left(\frac{1}{\alpha^{\prime}} \frac{\min (a, 1)}{\min \left(a^{\prime}, 1\right)}, 1\right) \\
+o\left(\frac{1}{n b_{n}}\right)+O\left(\frac{\log n}{n}\right)+O\left(\frac{\log ^{2} n}{n^{2} b_{n}^{2}}\right) & \left.\left.\times \frac{\left.a^{\prime}, 1\right)}{a^{\prime} \alpha}\right]\right\}
\end{aligned}
$$

if $n \rightarrow \infty, b_{n} \downarrow 0, n b_{n} \rightarrow \infty$.

In Theorem 5.3 the covariance properties of the smoothed spectral estimates are given. Thus Theorems 5.2 and 5.3 show that the smoothed spectral estimates give consistent estimates of the spectral mass on the lines of concentration if there is no aliasing. The possible effects of aliasing can be read off from Theorem 5.2. Also consider formula (5.8) for the case of the variance when there is no aliasing. Then, except for a finite number of points, the expression on the right-hand side is

$$
\frac{2 \pi}{(n+1) b_{n}} f_{1,0}(\eta+w) f_{1,0}(-\eta) \int K(x)^{2} d x
$$

the contribution from the diagonal $a=1, b=0$. The additional terms that can arise at this finite number of points correspond to the doubling at $0, \pi$ in the case of spectral estimates for a stationary process.

If the process is almost periodically correlated, all the spectral lines have slope $1, a=a^{\prime}=\alpha=\alpha^{\prime}=1$. The following corollary is an immediate consequence of the theorem. 
COROLLARY 5.1. If the process is almost periodically correlated, then, under the conditions of Theorem 5.3,

$$
\begin{aligned}
& \operatorname{cov}\left(\hat{f}_{1, w}(\eta), \hat{f}_{1, w^{\prime}}\left(\eta^{\prime}\right)\right) \\
&=\frac{2 \pi}{(n+1) b_{n}} \sum_{\substack{k^{\prime}, k \\
b, b^{\prime}}}\left[\delta_{\left(\left(\eta^{\prime}-\eta+w^{\prime}-w+b\right) \bmod 2 \pi\right), 0} \delta_{\left(\left(\eta-\eta^{\prime}+b^{\prime}\right) \bmod 2 \pi\right), 0}\right. \\
& \quad \times f_{1, b}\left(\eta^{\prime}+w^{\prime}+2 \pi k\right) f_{1, b^{\prime}}\left(-\eta^{\prime}+2 \pi k^{\prime}\right) \int K(x)^{2} d x \\
&+ \delta_{\left(\left(-\eta^{\prime}+b-\eta-w\right) \bmod 2 \pi\right), 0} \delta_{\left(\left(\eta^{\prime}+w^{\prime}+\eta+b^{\prime}\right) \bmod 2 \pi\right), 0} \\
& \times f_{1, b}\left(-\eta^{\prime}+2 \pi k\right) f_{1, b^{\prime}}\left(\eta^{\prime}+w^{\prime}+2 \pi k^{\prime}\right) \\
&\left.\times \int K(-x) K(x) d x\right] \\
&+o\left(\frac{1}{n b_{n}}\right)+O\left(\frac{\log n}{n}\right)+O\left(\frac{\log ^{2} n}{n^{2} b_{n}^{2}}\right) .
\end{aligned}
$$

Now we discuss briefly the estimation of the cross-spectrum of harmonizable processes.

DEFINITION. Stochastic processes $\left\{X^{(j)}(t) \mid j=1, \ldots, J\right\}$ are jointly harmonizable if, for $l, j=1, \ldots, J$,

$$
X_{t}^{(j)} \equiv X^{(j)}(t)=\int e^{i t \lambda} d Z_{j}(\lambda)
$$

and

$$
\begin{aligned}
\operatorname{cov}\left(X^{(l)}(t), X^{(j)}(\tau)\right) & =\iint_{-\infty}^{\infty} e^{i t \lambda-i \tau \mu} \operatorname{cov}\left(d Z_{l}(\lambda), d Z_{j}(\mu)\right) \\
& =\iint_{-\infty}^{\infty} e^{i t \lambda-i \tau \mu} d H_{l, j}(\lambda, \mu)
\end{aligned}
$$

with

$$
\iint_{-\infty}^{\infty}\left|d H_{l, j}(\lambda, \mu)\right| d \lambda d \mu<\infty
$$

If cross-spectral mass is concentrated on lines in the set

$$
\mathcal{L}=\{(a, b) \mid \lambda=a \mu+b\}
$$

and spectral densities exist, then

$$
d H_{i, j}(\lambda, \mu)=\sum_{(a, b) \in \mathscr{L}} \delta_{\lambda-a \mu-b, 0} f_{a, b}^{(i, j)}(\mu) d \mu .
$$


An obvious periodogram is

$$
I_{l, n}^{(i, j)}(\lambda, \mu)=\frac{1}{2 \pi n} F_{l, n}^{(i)}(\lambda) \overline{F_{l, n}^{(j)}(\mu)},
$$

with

$$
F_{l, n}^{(k)}(\lambda)=\sum_{t=0}^{n-1} X_{t+l J}^{(k)} e^{-i(t+l J) \lambda} .
$$

Under assumptions similar to Assumptions 1-3 analogs of the previous results in the univariate case hold for the multivariate case.

6. Simulation examples. In this section we give an example of a harmonizable process with spectral support on lines and carry out spectral density estimation. Let

$$
Y_{t}=\int e^{i t \lambda} d Z(\lambda)
$$

be a stationary process with spectral density $f(\cdot)$ and spectral distribution $F(\cdot)$. Let

$$
X_{t}=Y_{t}+\cos (w t) Y_{\alpha t},
$$

with $w, \alpha \in(0, \infty)$. This is the multipath problem of (2.5) with $k=1$ where we have made the example more complicated by including a "carrier" $\cos (w t)$ for the Doppler stretched signal. Then

$$
\begin{aligned}
X_{t} & =\int e^{i t \lambda} d Z(\lambda)+\frac{1}{2} \int\left(e^{i w t}+e^{-i w t}\right) e^{i \alpha t \lambda} d Z(\lambda) \\
& =\int e^{i t \lambda} d Z(\lambda)+\frac{1}{2} \int e^{i t \lambda}\left[d Z\left(\frac{\lambda-w}{\alpha}\right)+d Z\left(\frac{\lambda+w}{\alpha}\right)\right] \\
& =\int e^{i t \lambda} d Z_{1}(\lambda),
\end{aligned}
$$

with $d Z_{1}(\lambda)=d Z(\lambda)+\frac{1}{2} d Z\left(\frac{1}{\alpha}(\lambda-w)\right)+\frac{1}{2} d Z\left(\frac{1}{\alpha}(\lambda+w)\right)$. Then the spectrum of $X_{t}$ is given by $d H(\lambda, \mu) \equiv E d Z_{1}(\lambda) \overline{d Z_{1}(\mu)}$. Using the orthogonal increment property of the $Z(\lambda)$ process and the fact that $E d Z(\lambda) \overline{d Z(\lambda)}=f(\lambda) d \lambda$, we have

$$
E d Z(\lambda) \overline{d Z(\mu)}=f(\mu) \delta_{\lambda, \mu} d \mu
$$

$$
\begin{aligned}
E \frac{1}{2} d Z\left(\frac{1}{\alpha}(\lambda-w)\right) \frac{1}{2} \overline{d Z\left(\frac{1}{\alpha}(\mu-w)\right)} \\
\quad=\frac{1}{4} E\left\{\lim _{h \rightarrow 0} \frac{1}{h}\left|Z\left(\frac{1}{\alpha}(\mu+h-w)\right)-Z\left(\frac{1}{\alpha}(\mu-w)\right)\right|^{2} \delta_{\lambda, \mu} d \mu\right\}
\end{aligned}
$$




$$
\begin{aligned}
& =\frac{1}{4} \lim _{h \rightarrow 0} \frac{1}{h}\left[F\left(\frac{1}{\alpha}(\mu-w)+\frac{h}{\alpha}\right)-F\left(\frac{1}{\alpha}(\mu-w)\right)\right] \delta_{\lambda, \mu} d \mu \\
& =\frac{1}{4 \alpha} f\left(\frac{1}{\alpha}(\mu-w)\right) \delta_{\lambda, \mu} d \mu, \\
E \frac{1}{2} d Z & \left(\frac{1}{\alpha}(\lambda-w)\right) \overline{d Z(\mu)}=\frac{1}{2} E d Z(\mu) \overline{d Z(\mu)} \delta_{(1 / \alpha)(\lambda-w), \mu} d \mu \\
= & \frac{1}{2} f(\mu) \delta_{\lambda, \alpha \mu+w} d \mu, \\
E d Z & (\lambda) \frac{1}{2} d Z\left(\frac{1}{\alpha}(\mu-w)\right) \\
= & \frac{1}{2} E\left|d Z\left(\frac{1}{\alpha}(\mu-w)\right)\right|^{2} \delta_{\lambda,(1 / \alpha)(\mu-w)} d \mu \\
= & \frac{1}{2 \alpha} f\left(\frac{1}{\alpha}(\mu-w)\right) \delta_{\lambda,(1 / \alpha)(\mu-w)} d \mu,
\end{aligned}
$$

with corresponding expressions for all other possible terms. Therefore

$$
\begin{aligned}
d H(\lambda, \mu)= & {\left[f(\mu)+\frac{1}{4 \alpha} f\left(\frac{1}{\alpha}(\mu-w)\right)+\frac{1}{4 \alpha} f\left(\frac{1}{\alpha}(\mu+w)\right)\right] \delta_{\lambda, \mu} d \mu } \\
& +\frac{1}{2} f(\mu) \delta_{\lambda, \alpha \mu+w} d \mu+\frac{1}{2} f(\mu) \delta_{\lambda, \alpha \mu-w} d \mu \\
& +\frac{1}{2 \alpha} f\left(\frac{1}{\alpha}(\mu-w)\right) \delta_{\lambda,(1 / \alpha)(\mu-w)} d \mu \\
& +\frac{1}{2 \alpha} f\left(\frac{1}{\alpha}(\mu+w)\right) \delta_{\lambda,(1 / \alpha)(\mu+w)} d \mu \\
& +\frac{1}{4 \alpha} f\left(\frac{1}{\alpha}(\mu-w)\right) \delta_{\lambda, \mu-2 w} d \mu \\
& +\frac{1}{4 \alpha} f\left(\frac{1}{\alpha}(\mu+w)\right) \delta_{\lambda, \mu+2 w} d \mu .
\end{aligned}
$$

The spectral mass of $X_{t}$ is supported on the seven lines given by

$$
\lambda=\mu, \quad \lambda=\alpha \mu+w, \quad \lambda=\alpha \mu-w, \quad \lambda=\frac{1}{\alpha}(\mu-w),
$$

$$
\lambda=\frac{1}{\alpha}(\mu+w), \quad \lambda=\mu-2 w \quad \text { and } \quad \lambda=\mu+2 w,
$$

which we shall refer to as lines (1)-(7), respectively. These lines are shown in Figure 1 for $(\alpha, w)=(0.5,0.5)$. 


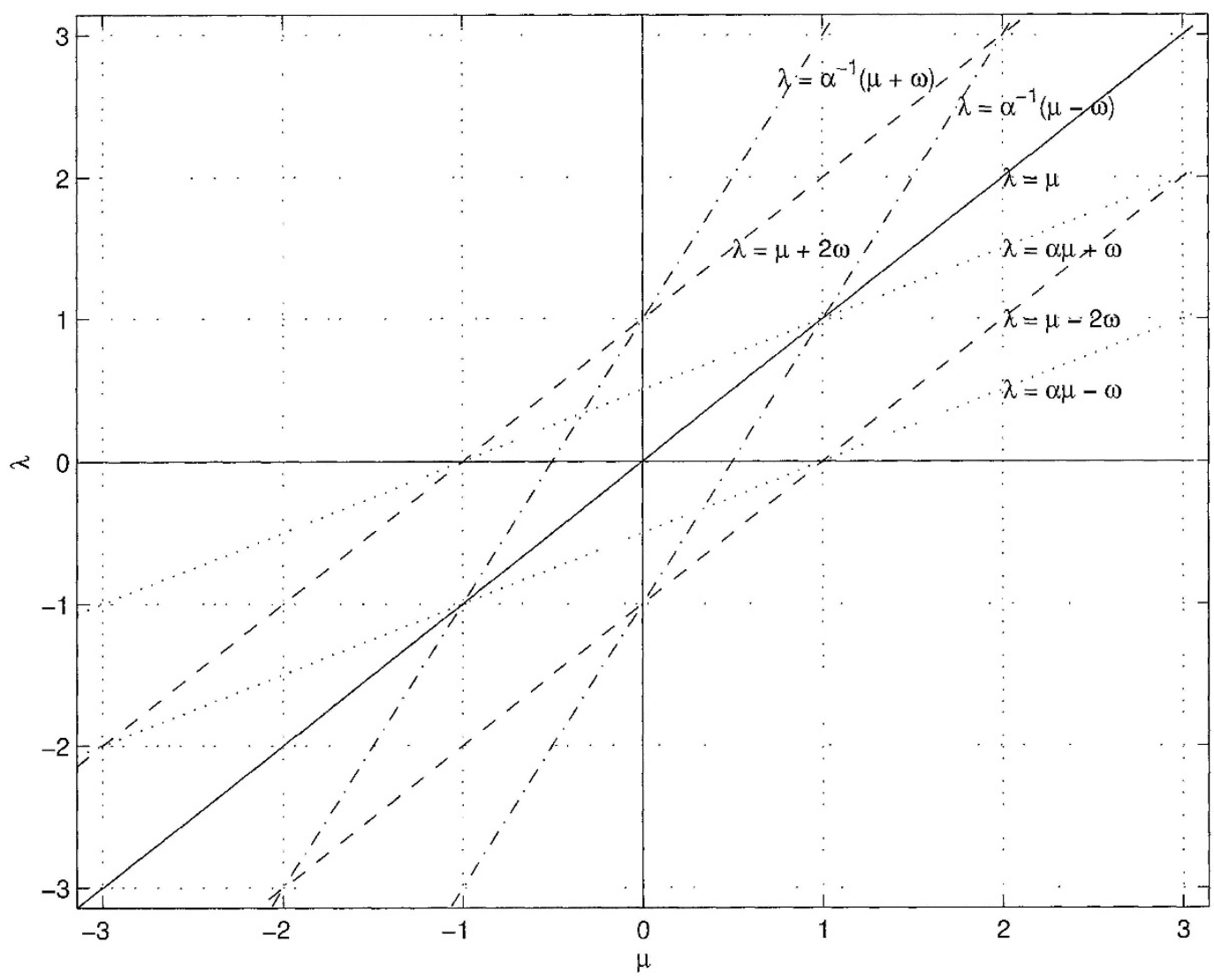

FIG. 1. Spectral support lines for $y_{t}=x_{t}+\cos (\omega t) x_{\alpha t}$ with $(\alpha, \omega)=(0.5,0.5)$.

As an example, consider

$$
Y_{t}=\varepsilon_{t}+0.5 \varepsilon_{t-1},
$$

with $\varepsilon_{t}$ i.i.d. $N(0,1)$.

Five thousand independent $Y_{t}$ with $t=-128, \ldots, 128$ are generated. Shannon interpolation is used to obtain $Y_{\alpha t}, t=-128, \ldots, 128$, for $\alpha=0.5$. Using (6.2), $X_{t}, t=-128, \ldots, 128$, is obtained. Periodograms are formed from (3.9) on each of the seven lines given by (6.4). Five thousand independent such periodograms are averaged and for each line both real and imaginary parts of the corresponding periodogram are plotted against the corresponding theoretical values given by (6.3). These plots are shown in Figure 2 in order starting from term 1. Relative to term 1 , the sum of the three terms which gives the theoretical spectral mass on the diagonal line [see line 1 from (6.3)] is plotted as a dashed curve. The averaged periodogram of $X_{t}$ is plotted with it as the solid line and we see that it is roughly unbiased with some variability. The theoretical spectral mass and its estimate are almost superimposed. The theoretical spectral mass is also plotted as the dashed curve slightly below its estimate with an offset. The two spikes located at \pm 1 are 

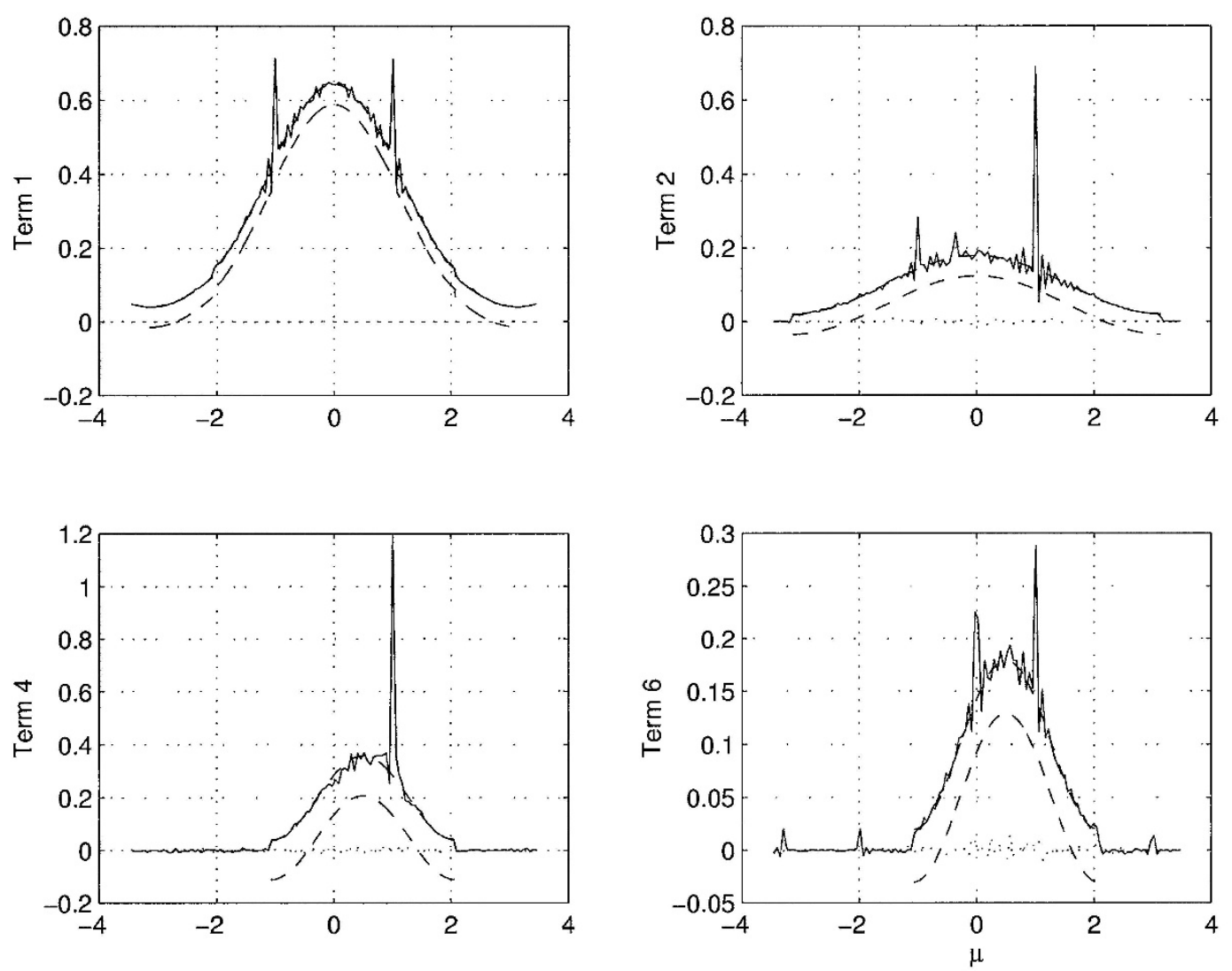

FIG. 2. Averaged periodogram spectral estimates on different lines; $(\alpha, \omega)=(0.5,0.5)$; sample size, 257, each averaged 5000 times; - , estimated real part; $\cdots$, estimated imaginary part; - .., theoretical real part; theoretical imaginary parts are all 0 for this example and are not plotted. Theoretical real parts are almost superimposed on their estimates so they are also plotted with an arbitrary offset.

due to the intersection of the diagonal line with two other spectral support lines. Lines 2 and 4 intersect line 1 at +1 while lines 3 and 5 intersect line 1 at -1 (see Figure 1). There are extra spectral masses contributing to the diagonal lines at these intersection points. Similar remarks can be made for all other graphs where there are spikes. These graphs are consistent with the properties of the periodogram given by (3.10) or (5.4). Since the theoretical spectral functions are almost the same as their estimates, we also plot a shift of the real part of the theoretical spectral functions to better indicate their character. Terms 2, 4 and 6 correspond to lines 2, 4 and 6 from (6.3). Terms 3, 5 and 7 are not plotted since they are symmetric versions of terms 2,4 and 6 , respectively. Overall, we see that the procedure is effective in estimating the spectral mass supported on lines. One way to remove the spikes is to use the average of neighboring spectral mass on the line. From the graphs in Figure 2, we also see the effects of the band-limitness of $Y_{t}$ [see (6.5)] in the sudden drop-offs. 
$f(\mu)$ on $\lambda=\mu$

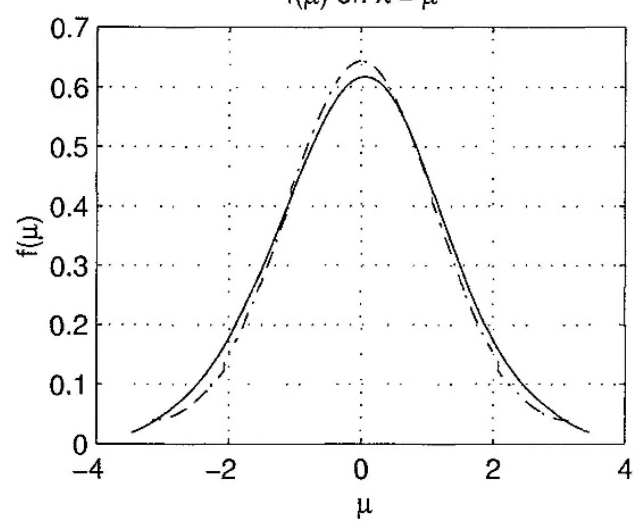

$f(\mu)$ on $\lambda=\alpha^{-1}(\mu-\omega)$

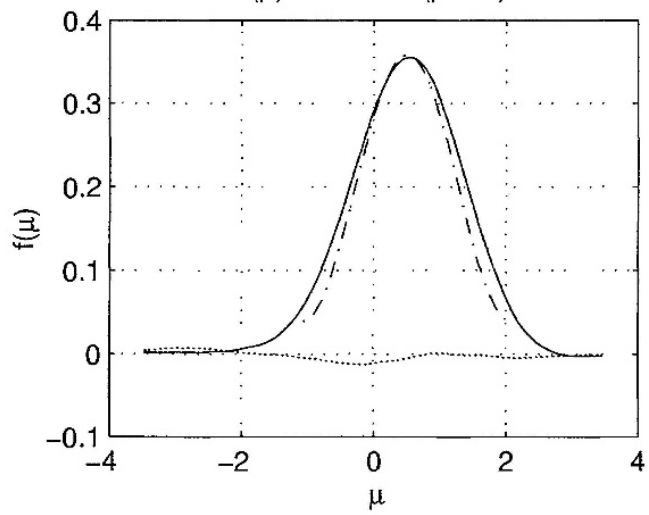

$\mathrm{f}(\mu)$ on $\lambda=\alpha \mu+\omega$

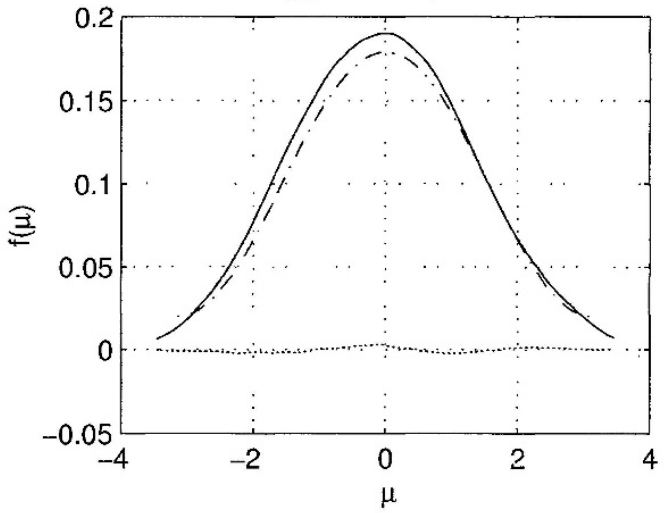

$f(\mu)$ on $\lambda=\mu-2 \omega$

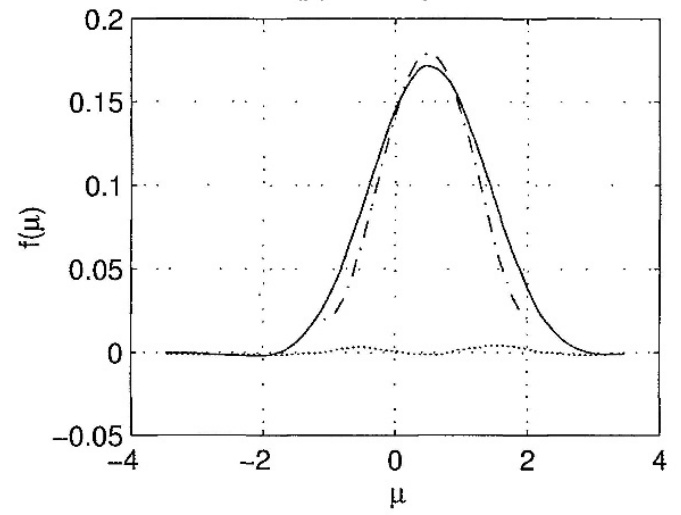

FIG. 3. Spectral estimates on different lines; $(\alpha, \omega)=(0.5,0.5) ;$ sample size, $16385 ;-$, estimated real part; $\cdots$, estimated imaginary part; - -, theoretical real part; theoretical imaginary parts are all 0 for this example and are not plotted.

In reality, we do not have independent realizations with which to average the periodograms. Using the same model, we generated one realization of $X_{t}$ for $t=-2^{13}, \ldots, 2^{13}$. Then a smoothed periodogram of the form in (5.2) is used to estimate the spectral mass on each of the support lines. Results are given in Figure 3.

Symmetry properties given in (2.5) make some graphs redundant. Consequently, some graphs are not plotted. We see that the estimates are generally very close to the theoretical ones. These computations confirm the results of Theorems 5.2 and 5.3. Spikes at the intersection of spectral support lines (see Figure 2) are removed. The values at such intersection points are determined by the direction of smoothing. If the lines of spectral support are not known a priori, the results of Theorems 5.2 and 5.3 imply that one can search for these using a grid of points, by computing smoothed periodograms in a grid of directions at each of these points and recording the largest magnitude among these directions at each point. Set an appropriate threshold level to remove all those points whose spectral 
magnitude is below the threshold. Then by connecting the remaining points one obtains information about the locations of the spectral support lines. Alternatively, one can search for $(\alpha, w)$ in that if certain statistics from the spectral estimate along the line represented by $(\alpha, w)$ are significantly different from 0 then $(\alpha, w)$ represents a line of spectral support. Spectral mass on these lines of spectral support can be estimated. The spectral function $f(\cdot)$ of the unobserved signal $Y(t)$ can be estimated from estimates of off-diagonal spectral lines in (6.3). These are obviously computationally intensive and are not pursued here.

7. Proofs. We need the following lemmas in the derivations of the results and they are used often.

LEMMA 7.1. Let

$$
D_{n}(x)=\sum_{t=0}^{n-1} e^{i t x}=\frac{e^{i n x}-1}{e^{i x}-1}
$$

and

$$
\tilde{D}_{n}(x)=\frac{e^{i n x}-1}{i x}
$$

Then $D_{n}^{\prime}(x)=D_{n}(x)-\tilde{D}_{n}(x)$ is uniformly bounded in $[-2 \pi+c, 2 \pi-c]$ for a fixed $c>0$. In particular, $D_{n}^{\prime}(x)$ is uniformly bounded in $[-\pi, \pi]$ with

$$
D_{n}^{\prime}(x)=\left(e^{i n x}-1\right) \frac{i x-e^{i x}+1}{\left(e^{i x}-1\right) i x} .
$$

The derivation is straightforward.

LEMMA 7.2. For any real $a,|a|>0,|y| \leq \pi$,

$$
\int_{-\pi}^{\pi} D_{n}(a x+y) D_{n}(x) d x=\int_{-\pi}^{\pi} \tilde{D}_{n}(a x+y) \tilde{D}_{n}(x) d x+O(\log n) .
$$

PROOF. Since $D_{n}(x)=D_{n}^{\prime}(x)+\tilde{D}_{n}(x)$,

$$
\begin{aligned}
\int_{-\pi}^{\pi} D_{n}(a x+y) D_{n}(x) d x-\int_{-\pi}^{\pi} \tilde{D}_{n}(a x+y) \tilde{D}_{n}(x) d x \\
=\int_{-\pi}^{\pi} D_{n}^{\prime}(a x+y) D_{n}^{\prime}(x) d x+\int_{-\pi}^{\pi} D_{n}^{\prime}(a x+y) \tilde{D}_{n}(x) d x \\
\quad+\int_{-\pi}^{\pi} \tilde{D}_{n}(a x+y) D_{n}^{\prime}(x) d x=(1)+(2)+(3) .
\end{aligned}
$$


We first note that, for a fixed $M>0$,

$$
\begin{aligned}
\int_{-M}^{M}\left|\tilde{D}_{n}(x)\right| d x & =\int_{|x| \leq 1 / n^{2}}\left|\tilde{D}_{n}(x)\right| d x+\int_{M \geq|x| \geq 1 / n^{2}}\left|\tilde{D}_{n}(x)\right| d x \\
& \leq \frac{2}{n}+O(\log n)+O(\log M)=O(\log n) .
\end{aligned}
$$

Using Lemma 7.1, for a constant $C^{\circ}$,

$$
|(1)| \leq C^{\circ} \int_{-\pi}^{\pi}\left|D_{n}^{\prime}(a x+y)\right| d x=\frac{C^{\circ}}{|a|} \int_{-a \pi+y}^{a \pi+y}\left|D_{n}^{\prime}(x)\right| d x .
$$

For a fixed $d \in\left[d^{\prime}, \pi\right)$ with $d^{\prime}>0$, by Lemma 7.1,

$$
\int_{-d}^{d}\left|D_{n}^{\prime}(x)\right| d x=O(1)
$$

For $k \neq 0$,

$$
\begin{aligned}
\int_{2 k \pi-d}^{2 k \pi+d}\left|D_{n}^{\prime}(x)\right| d x & =\int_{2 k \pi-d}^{2 \pi k+d}\left|\frac{e^{i n x}-1}{e^{i x}-1}\right|\left|\frac{i x-e^{i x}+1}{i x}\right| d x \\
& \leq\left(1+\frac{2}{\pi}\right) \int_{2 k \pi-d}^{2 k \pi+d}\left|\frac{\sin \frac{n}{2} x}{\sin \frac{1}{2} x}\right| d x \\
& =\left(1+\frac{2}{\pi}\right) \int_{-d}^{d}\left|\frac{\sin \frac{n}{2} x}{\sin \frac{1}{2} x}\right| d x \leq O(\log n) .
\end{aligned}
$$

It is clear that $\int_{A}\left|D_{n}^{\prime}(x)\right| d x=O(1)$ for a closed interval $A$ not containing $2 \pi k$ for integer $k$. Therefore $|(1)|=O(\log n)$. A similar argument applied to $\tilde{D}_{n}(a x+y)$ shows that $|(3)|=O(\log n)$. Finally,

$$
|(2)|=\int_{|x| \leq d}\left|D_{n}^{\prime}(a x+y) \tilde{D}_{n}(x)\right| d x+\int_{\pi \geq|x| \geq d}\left|D_{n}^{\prime}(a x+y) \tilde{D}_{n}(x)\right| d x=(4)+(5) .
$$

For fixed $a$, we can choose a $d$ such that $|a x+y| \leq 2 \pi-c$ for a fixed $c>0$ for all $|x| \leq d$ and therefore $D_{n}^{\prime}(a x+y)$ is uniformly bounded by Lemma 7.1. Hence

$$
\text { (4) } \leq C^{\circ} \int_{|x| \leq d}\left|\tilde{D}_{n}(x)\right| d x=O(\log n) .
$$

For the same $d,\left|\tilde{D}_{n}(x)\right| \leq 2 d^{-1}$ for $\pi \geq|x| \geq d$. Hence

$$
\text { (5) } \leq 2 d^{-1} \int_{\pi \geq|x| \geq d}\left|D_{n}^{\prime}(a x+y)\right| d x=O(\log n) .
$$

This completes the proof of Lemma 7.2. 
Proof of Theorem 3.1. Given $\left\{X_{t}\right\}_{t=-N}^{N}, \lambda^{\prime}=\alpha \mu^{\prime}+w$. Let [using (3.1) and (3.2)]

$$
G_{0} \equiv E I_{l, n}\left(\lambda^{\prime}, \mu^{\prime}\right)=\frac{1}{2 \pi n} \iint_{-\infty}^{\infty} \sum_{t, \tau=0}^{n-1} e^{i t\left(\lambda-\lambda^{\prime}\right)-i \tau\left(\mu-\mu^{\prime}\right)}
$$

$$
=e^{-i l J\left(\lambda^{\prime}-\mu^{\prime}\right)} \sum_{(a, b) \in \mathcal{L}} \sum_{k} e^{i l J(\lambda-\mu)} d H(\lambda, \mu) e^{-i l J\left(\lambda^{\prime}-\mu^{\prime}\right)}
$$

where

$$
\begin{aligned}
G_{1}(k, a, b) \equiv G_{1}\left(k, a, b, \lambda^{\prime}, \mu^{\prime} ; n, l, J\right) \equiv G_{1} \\
\equiv \frac{1}{2 \pi n} \int_{\mu^{\prime}-\pi+2 k \pi}^{\mu^{\prime}+\pi+2 k \pi} D_{n}\left(\lambda-\lambda^{\prime}\right) D_{n}\left(-\mu+\mu^{\prime}\right) e^{i l J(\lambda-\mu)} \\
\times f_{a, b}(\mu) \delta_{\lambda, a \mu+b} d \mu .
\end{aligned}
$$

Let $x=\mu-\mu^{\prime}-2 \pi k, \mu=x+\mu^{\prime}+2 \pi k$. Then, using $\lambda=a \mu+b$ and $\lambda^{\prime}=\alpha \mu^{\prime}+w$,

$$
\begin{aligned}
G_{1}=\frac{1}{2 \pi n} \int_{-\pi}^{\pi} & D_{n}\left(a x+(a-\alpha) \mu^{\prime}+b-w+2 \pi k a\right) D_{n}(-x) \\
& \times e^{i l J(a-1) x} f_{a, b}\left(x+\mu^{\prime}+2 \pi k\right) d x e^{i l J\left[b+(a-1)\left(\mu^{\prime}+2 \pi k\right)\right]}
\end{aligned}
$$

since

$$
\begin{gathered}
-\mu+\mu^{\prime}=-x-2 \pi k, \quad D_{n}\left(-\mu+\mu^{\prime}\right)=D_{n}(-x-2 \pi k)=D_{n}(-x), \\
\lambda-\mu=(a-1) x+b+(a-1)\left(\mu^{\prime}+2 \pi k\right) .
\end{gathered}
$$

Notice that $e^{-i l J\left(\lambda^{\prime}-\mu^{\prime}\right)} e^{i l J\left[b+(a-1)\left(\mu^{\prime}+2 \pi k\right)\right]}=\exp \left\{i l J\left(2 \pi k a+(a-\alpha) \mu^{\prime}+b-\right.\right.$ $w)\}$. Then, using (3.4),

$$
G_{0}=\sum_{(a, b) \in \mathcal{L}} \sum_{k} e^{i l J \cdot y(k, a, b ; \lambda, \mu)} G_{2},
$$

with

$$
G_{2}=\frac{1}{2 \pi n} \int_{-\pi}^{\pi} D_{n}(a x+y) D_{n}(-x) e^{i l J(a-1) x} f_{a, b}\left(x+\mu^{\prime}+2 \pi k\right) d x .
$$

Because of the continuous differentiability of $f_{a, b}$, we have

$$
f_{a, b}\left(x+\mu^{\prime}+2 \pi k\right)=f_{a, b}\left(\mu^{\prime}+2 \pi k\right)+x f_{a, b}^{\prime}\left(x^{*}+2 \pi k\right),
$$

with $x^{*}$ between $\mu^{\prime}$ and $\mu^{\prime}+x$. Then

$$
G_{2}=G_{3}+G_{4},
$$


with

$$
G_{4}=\frac{1}{2 \pi n} \int_{-\pi}^{\pi} D_{n}(a x+y) D_{n}(-x) x e^{i l J(a-1) x} f_{a, b}^{\prime}\left(x^{*}+2 \pi k\right) d x,
$$

$$
\left|G_{4}\right| \leq g(2 \pi k) \cdot O\left(\frac{\log n}{n}\right)
$$

by Assumption 2 and

$$
\begin{array}{r}
G_{3}=f_{a, b}\left(\mu^{\prime}+2 \pi k\right) \frac{1}{2 \pi n} \int_{-\pi}^{\pi} D_{n}(a x+y) D_{n}(-x) e^{i l J(a-1) x} d x \\
=f_{a, b}\left(\mu^{\prime}+2 \pi k\right)\left[\frac{1}{2 \pi n} \int_{-\pi}^{\pi} \tilde{D}_{n}(a x+y) \tilde{D}_{n}(-x) e^{i l J(a-1) x} d x\right. \\
\left.+O\left(\frac{\log n}{n}\right)\right]
\end{array}
$$

using Lemma 7.2. Consider

$$
G_{5}=\frac{1}{2 \pi n} \int_{-\pi}^{\pi} \frac{e^{i n(a x+y)}-1}{i(a x+y)} \frac{e^{-i n x}-1}{-i x} e^{i l J(a-1) x} d x .
$$

Let $n x=z$. Then

$$
\begin{aligned}
G_{5} & =\frac{1}{2 \pi n} \int_{-n \pi}^{n \pi} \frac{e^{i(a z+n y)}-1}{i(a z+n y) / n} \frac{e^{-i z}-1}{-i z / n} e^{i l J(a-1) z / n} d z / n \\
& =\frac{1}{2 \pi} \int_{-\infty}^{\infty} \frac{e^{i(a z+n y)}-1}{i(a z+n y)} \frac{e^{-i z}-1}{-i z} e^{i l J(a-1) z / n} d z
\end{aligned}
$$

$$
\begin{aligned}
& -\frac{1}{2 \pi} \int_{|z|>n \pi} \frac{e^{i(a z+n y)}-1}{i(a z+n y)} \frac{e^{-i z}-1}{-i z} e^{i l J(a-1) z / n} d z \\
= & G_{6}-G_{7} .
\end{aligned}
$$

To evaluate the second term $G_{7}$, we first note that $|y| \leq \pi$ and

$$
\begin{aligned}
& \int_{|z| \geq n \pi}\left|\frac{e^{i(a z+n y)}-1}{i(a z+n y)} \frac{e^{-i z}-1}{-i z}\right| d z \\
& \quad \leq \frac{1}{|a|}\left[\int_{z \in A_{1} \cap A}+\int_{z \in A_{1}^{c} \cap A_{2}}+\int_{|z| \geq n \pi+|n \pi / a|}\right]\left|\frac{e^{i(a z+n y)}-1}{i(z+n y / a)} \frac{e^{-i z}-1}{-i z}\right| d z,
\end{aligned}
$$

where $A_{1}=[-n y / a-c,-n y / a+c]$ with fixed $c>0$ and $A=\{z|| z \mid \geq n \pi\}$, $A_{2}=\{z|n \pi \leq| z|\leq n \pi+| n \pi / a \mid\}$. For the first integral, $z \in A_{1} \cap A$ and $\left|\left(e^{i(a z+n y)}-1\right) /(i(z+n y / a))\right| \leq|a|,\left|\left(e^{-i z}-1\right) /(-i z)\right| \leq 1 / n$. Therefore

$$
\int_{z \in A_{1} \cap A}\left|\frac{e^{i(a z+n y)}-1}{i(z+n y / a)} \frac{e^{-i z}-1}{-i z} d z\right| \leq \frac{2|a| c}{n}=O\left(\frac{1}{n}\right) .
$$


The second integral is bounded by

$$
\frac{1}{n \pi} \int_{z \in A_{1}^{c} \cap A_{2}}\left|\frac{1}{z+n y / a}\right| d z=O\left(\frac{\log n}{n}\right) .
$$

The third integral is bounded by

$$
C \int_{|z|>n \pi} \frac{1}{z^{2}} d z=O\left(\frac{1}{n}\right) .
$$

From these we see

$$
G_{7}=\frac{1}{2 \pi} \int_{|z|>n \pi} \frac{e^{i(a z+n y)}-1}{i(a z+n y)} \frac{e^{-i z}-1}{-i z} e^{i l J(a-1) z / n} d z=O\left(\frac{\log n}{n}\right)
$$

To evaluate $G_{6}$, we note that

$$
\begin{gathered}
\frac{e^{i(a z+n y)}-1}{i(a z+n y)}=\int_{-\infty}^{\infty} \frac{1}{a} 1_{[0, a]}(t) e^{i n y t / a} e^{i z t} d t \\
{\left[\frac{e^{-i z}-1}{-i z} e^{i l J(a-1) z / n}\right]^{*}=\int_{-\infty}^{\infty} 1_{[-l J(a-1) / n, 1-l J(a-1) / n]}(t) e^{i z t} d t}
\end{gathered}
$$

and, using the Parseval relation, we have

$$
\begin{aligned}
G_{6} & =\frac{1}{a} \int_{-\infty}^{\infty} 1_{(0, a]}(t) 1_{[-l J(a-1) / n, 1-l J(a-1) / n]}(t) e^{i n y t / a} d t \\
& =\left\{\begin{array}{c}
\frac{1}{i n y}\left[\exp \left(i \frac{n y}{a}\left[\min \left(a, 1-\frac{l J(a-1)}{n}\right)\right]\right)\right. \\
\left.-\exp \left(i \frac{n y}{a}\left[\max \left(0, \frac{-l J(a-1)}{n}\right)\right]\right)\right] \\
\text { if }[0, a] \cap\left[\frac{-l J(a-1)}{n}, 1-\frac{l J(a-1)}{n}\right] \neq \varnothing, \\
0, \quad \text { otherwise. }
\end{array}\right.
\end{aligned}
$$

Successively using (7.2)-(7.9) and (7.12), we obtain Theorem 3.1.

PROOF OF THEOREM 4.1. Let $d_{n}=1 /(2 \pi n)$. Then

$$
\begin{gathered}
\operatorname{cov}\left(I_{l, n}(\lambda, \mu), I_{l, n}\left(\lambda^{\prime}, \mu^{\prime}\right)\right) \\
=d_{n}^{2} \sum_{t, \tau, t^{\prime}, \tau^{\prime}=0}^{n}\left[r_{t+l J, t^{\prime}+l J} r_{\tau+l J, \tau^{\prime}+l J}+r_{t+l J, \tau^{\prime}+l J} r_{\tau+l J, t^{\prime}+l J}\right] \\
\times \exp \left\{i\left[-(t+l J) \lambda+(\tau+l J) \mu+\left(t^{\prime}+l J\right) \lambda^{\prime}-\left(\tau^{\prime}+l J\right) \mu^{\prime}\right]\right\} \\
=d_{n}^{2} \sum_{t, \tau, t^{\prime}, \tau^{\prime}=0}^{n}\left[E \int e^{i(t+l J) u} d Z(u) \int e^{-i\left(t^{\prime}+l J\right) v} \overline{d Z(v)}\right. \\
\times E \int e^{i(\tau+l J) r} d Z(r) \int e^{-i\left(\tau^{\prime}+l J\right) s} \overline{d Z(s)}
\end{gathered}
$$




$$
\begin{aligned}
& +E \int e^{i(t+l J) u} d Z(u) \int e^{-i\left(\tau^{\prime}+l J\right) v} \overline{d Z(v)} \\
& \left.\times E \int e^{i(\tau+l J) r} d Z(r) \int e^{-i\left(t^{\prime}+l J\right) s} \overline{d Z(s)}\right] \\
& \times \exp \left\{i\left[-(t+l J) \lambda+(\tau+l J) \mu+\left(t^{\prime}+l J\right) \lambda^{\prime}-\left(\tau^{\prime}+l J\right) \mu^{\prime}\right]\right\} \\
& =d_{n}^{2} \iiint \int \sum_{t, \tau, t^{\prime}, \tau^{\prime}=0}^{n}\left[\operatorname { e x p } \left\{i \left[t(u-\lambda)+\tau(r+\mu)+t^{\prime}\left(\lambda^{\prime}-v\right)\right.\right.\right. \\
& \left.\left.-\tau^{\prime}\left(\mu^{\prime}+s\right)\right]\right\} \exp \{i l J[u-v+r-s]\} \\
& +\exp \left\{i \left[t(u-\lambda)+\tau(r+\mu)+t^{\prime}\left(\lambda^{\prime}-s\right)\right.\right. \\
& \left.\left.-\tau^{\prime}\left(\mu^{\prime}+v\right)\right]\right\} \\
& \times \exp \{i l J[u-v+r-s]\}] \\
& \times \exp \left\{i l J\left[-\lambda+\mu+\lambda^{\prime}-\mu^{\prime}\right]\right\} d H(u, v) d H(r, s) \\
& =d_{n}^{2} \iiint \int\left[D_{n}(u-\lambda) D_{n}\left(\lambda^{\prime}-v\right) D_{n}(\mu+r) D_{n}\left(-\mu^{\prime}-s\right)\right. \\
& \times \exp \{i l J[u-v+r-s]\} \\
& +D_{n}(u-\lambda) D_{n}\left(-\mu^{\prime}-v\right) D_{n}(\mu+r) D_{n}\left(\lambda^{\prime}-s\right) \\
& \times \exp \{i l J(u-v+r-s)\}] \\
& \times \exp \left\{i l J\left(-\lambda+\mu+\lambda^{\prime}-\mu^{\prime}\right)\right\} d H(u, v) d H(r, s) \\
& =c_{1}+c_{2} \text {, }
\end{aligned}
$$

where

$$
\begin{array}{r}
c_{1}=\left\{\left[d_{n} \sum_{(a, b) \in \mathcal{L}} \int D_{n}(a v+b-\alpha \mu-w) D_{n}\left(\alpha^{\prime} \mu^{\prime}+w^{\prime}-v\right) f_{a, b}(v)\right.\right. \\
\left.\times \exp \{i l J(a v+b-v)\} d v] \exp \left\{-i l J\left(\lambda-\lambda^{\prime}\right)\right\}\right\} \\
\times\left\{\left[d_{n} \sum_{\left(a^{\prime}, b^{\prime}\right) \in \mathcal{L}} \int D_{n}\left(\mu+a^{\prime} s+b^{\prime}\right) D_{n}\left(-\mu^{\prime}-s\right) f_{a^{\prime}, b^{\prime}}(s)\right.\right. \\
\left.\left.\times \exp \left\{i l J\left(a^{\prime} s+b^{\prime}-s\right)\right\} d s\right] \exp \left\{-i l J\left(\mu^{\prime}-\mu\right)\right\}\right\},
\end{array}
$$




$$
\begin{aligned}
c_{2}=\left\{\left[d_{n} \sum_{(a, b) \in \mathcal{L}} \int D_{n}(a v+b-\alpha \mu-w) D_{n}\left(-\mu^{\prime}-v\right) f_{a, b}(v)\right.\right. \\
\left.\times \exp \{i l J(a v+b-v)\} d v] \exp \left\{-i l J\left(\lambda-\lambda^{\prime}\right)\right\}\right\} \\
\times\left\{\left[d_{n} \sum_{(a, b) \in \mathcal{L}} \int D_{n}\left(\mu+a^{\prime} s+b^{\prime}\right) D_{n}\left(\alpha^{\prime} \mu^{\prime}+w^{\prime}-s\right) f_{a^{\prime}, b^{\prime}}(s)\right.\right. \\
\left.\left.\times \exp \left\{i l J\left(a^{\prime} s+b-s\right)\right\} d s\right] \exp \left\{-i l J\left(\mu^{\prime}-\mu\right)\right\}\right\},
\end{aligned}
$$

with $u=a v+b, r=a^{\prime} s+b^{\prime}, \lambda=\alpha \mu+w, \lambda^{\prime}=\alpha^{\prime} \mu^{\prime}+w^{\prime}$. Now each term in the outer brackets has exactly the same form as the expectation of the periodogram [see (7.2)]. Therefore the covariance of the periodogram has the form of the product of the expectations of the periodogram.

Proof OF TheOREM 5.2. We now take the symmetrized version. From (5.7), (5.3), (5.5) and (3.4),

$$
\begin{aligned}
E \hat{f}_{\alpha, w}(\eta)= & \sum_{(a, b) \in \mathcal{L}} \sum_{k} \int f_{a, b}(\mu+2 \pi k) \operatorname{sinc}(a, y) K_{n}(\mu-\eta) d \mu \\
& +O\left(\frac{\log n}{n}\right) .
\end{aligned}
$$

Let

$$
\begin{aligned}
& E_{0} \equiv \int_{-\infty}^{\infty} f_{a, b}(\mu+2 \pi k) \operatorname{sinc}(a, y) K_{n}(\mu-\eta) d \mu \\
& =\int_{-\infty}^{\infty} f_{a, b}\left(\eta+b_{n} z+2 \pi k\right) \\
& \quad \times \operatorname{sinc}\left\{\frac{n+1}{2} l(a)[[2 \pi k a+(a-\alpha) \eta+b-w\right. \\
& \left.\left.\left.\quad+(a-\alpha) b_{n} z\right] \bmod 2 \pi\right]\right\} l(a) K(z) d z
\end{aligned}
$$

By Assumption 2, we have $f\left(\eta+b_{n} z+2 \pi k\right)=f(\eta+2 \pi k)+b_{n} z f_{a, b}^{\prime}\left(\eta_{k, z}^{*}+2 \pi k\right)$ for $\eta_{k, z}^{*}$ between $\eta+2 \pi k$ and $\eta+2 \pi k+b_{n} z$. Then $E_{0}=E_{1}+E_{2}$ with

$$
\begin{aligned}
& E_{1}=l(a) f_{a, b}(\eta+2 \pi k) E_{3}, \\
& E_{2}=b_{n} \int z f_{a, b}^{\prime}\left(\eta_{k, z}^{*}+2 \pi k\right) \\
& \quad \quad \times \operatorname{sinc}\left\{\frac{n+1}{2} l(a)\left[\left[y_{\eta}+(a-\alpha) b_{n} z\right] \bmod 2 \pi\right]\right\} l(a) K(z) d z, \\
& E_{3}=\int \operatorname{sinc}\left\{\frac{n+1}{2} l(a)\left[\left[y_{\eta}+(a-\alpha) b_{n} z\right] \bmod 2 \pi\right]\right\} K(z) d z
\end{aligned}
$$


and $y_{\eta} \equiv 2 \pi k a+(a-\alpha) \eta+b-w$. We evaluate $E_{2}$ first and then $E_{3}$.

$$
\begin{aligned}
E_{2}=b_{n} l(a) f_{a, b}^{\prime}(\eta+ & 2 \pi k) \int_{-c}^{c} z K(z) \\
& \times \operatorname{sinc}\left\{\frac{n+1}{2} l(a)\left[\left[y_{\eta}+(a-\alpha) b_{n} z\right] \bmod 2 \pi\right]\right\} d z+o\left(b_{n}\right)
\end{aligned}
$$

by the continuity of $f^{\prime}$ with $[-c, c]$ the support of $K$ for a finite $c$. For $a=\alpha$,

$$
\begin{aligned}
E_{2} & =b_{n} l(a) f_{a, b}^{\prime}(\eta+2 \pi k) \operatorname{sinc}\left\{\frac{n+1}{2} l(a)\left(y_{\eta} \bmod 2 \pi\right)\right\} \int_{-c}^{c} z K(z) d z+o\left(b_{n}\right) \\
& =o\left(b_{n}\right)
\end{aligned}
$$

by Assumption 3. For $a \neq \alpha, E_{2}=o\left(b_{n}\right)+E_{2}^{\prime}$ with

$$
\begin{aligned}
E_{2}^{\prime}= & b_{n} l(a) f_{a, b}^{\prime}(\eta+2 \pi k) \int_{-c}^{c} z K(z) \\
& \times \operatorname{sinc}\left\{\frac{n+1}{2} l(n)\left[\left[y_{\eta}+(a-\alpha) b_{n} z\right] \bmod 2 \pi\right]\right\} d z \\
= & b_{n} l(a) f_{a, b}^{\prime}(\eta+2 \pi k) \int_{-c}^{c} z K(z) \operatorname{sinc}\left\{\frac{n+1}{2} l(a)\left[\left(y_{\eta}\right)_{2 \pi}+(a-\alpha) b_{n} z\right]\right\} d z,
\end{aligned}
$$

where $\left(y_{\eta}\right)_{2 \pi}=\left(y_{\eta} \bmod 2 \pi\right)$. Therefore

$$
\begin{aligned}
\left|E_{2}^{\prime}\right| & \leq b_{n} C^{\circ} \int_{-c}^{c}\left|\operatorname{sinc}\left\{\frac{n+1}{2} l(a)\left[\left(y_{\eta}\right)_{2 \pi}+(a-\alpha) b_{n} z\right]\right\}\right| d z \\
& =C^{\circ} \int_{\left(y_{\eta}\right)_{2 \pi}-(a-\alpha) b_{n} c}^{\left(y_{\eta}\right)_{2 \pi}+(a-\alpha) b_{n} c}\left|\operatorname{sinc}\left\{\frac{n+1}{2} l(a) x\right\}\right| \frac{d x}{(a-\alpha)} \\
& \leq C^{\prime}\left[\int_{|x| \leq 1 / n}+\int_{C^{\prime \prime}>|x|>1 / n}\right]\left|\operatorname{sinc}\left\{\frac{n+1}{2} l(a) x\right\}\right| d x \\
& =O\left(\frac{1}{n}\right)+O\left(\frac{\log n}{n}\right),
\end{aligned}
$$

where $C^{\circ}, C^{\prime}$ are constants.

To evaluate $E_{3}$, we see that, by a similar argument, for $a \neq \alpha,\left|E_{3}\right|=O(\log n /$ $\left.n b_{n}\right)$.

If $a=\alpha$, then

$$
\begin{aligned}
E_{3} & =\int_{-c}^{c} \operatorname{sinc}\left\{\frac{n+1}{2} l(a)\left(y_{\eta} \bmod 2 \pi\right)\right\} K(z) d z \\
& =\operatorname{sinc}\left\{\frac{n+1}{2} l(a)\left(y_{\eta} \bmod 2 \pi\right)\right\} .
\end{aligned}
$$


Substituting these results on $E_{3}, E_{2}, E_{1}$ into $E_{0}$ using (7.13)-(7.16), we have the results.

Proof of THEOREM 5.3. We evaluate the covariance

$$
\begin{aligned}
C_{4} \equiv \operatorname{cov}\left(\hat{f}_{\alpha, w}(\eta), \hat{f}_{\alpha^{\prime}, w^{\prime}}\left(\eta^{\prime}\right)\right) \\
=\iint_{-\pi^{+}}^{\pi^{+}} K_{n}(\mu-\eta) K_{n}\left(\mu^{\prime}-\eta^{\prime}\right) \\
\quad \quad \times \operatorname{cov}\left(I_{n}(\alpha \mu+w, \mu), I_{n}\left(\alpha^{\prime} \mu^{\prime}+w^{\prime}, \mu^{\prime}\right)\right) d \mu d \mu^{\prime} \\
=C_{5}+C_{6}+O\left(\frac{\log n}{n}\right),
\end{aligned}
$$

with

$$
C_{5}=\iint_{-\pi^{+}}^{\pi^{+}} K_{n}(\mu-\eta) K_{n}\left(\mu^{\prime}-\eta^{\prime}\right) \Sigma^{1} \Sigma^{2} f_{a, b}\left(\lambda^{\prime}+2 \pi k\right) f_{a^{\prime}, b^{\prime}}\left(-\mu^{\prime}+2 \pi k^{\prime}\right)
$$

$$
\begin{gathered}
\times \operatorname{sinc}(a, y(1)) \operatorname{sinc}\left(a^{\prime}, y(2)\right) d \mu d \mu^{\prime}, \\
C_{6}=\iint_{\pi^{+}}^{\pi^{+}} K_{n}(\mu-\eta) K_{n}\left(\mu^{\prime}-\eta^{\prime}\right) \Sigma^{3} \Sigma^{4} f_{a, b}\left(-\mu^{\prime}+2 \pi k\right) f_{a^{\prime}, b^{\prime}}\left(\lambda^{\prime}+2 \pi k^{\prime}\right)
\end{gathered}
$$

$$
\times \operatorname{sinc}(a, y(3)) \operatorname{sinc}\left(a^{\prime}, y(4)\right) d \mu d \mu^{\prime}
$$

using (5.5). The sum $\Sigma^{1}$ is over $|y(1)|<v$ and $|y(2)| \leq v, \Sigma^{2}$ and $\Sigma^{4}$ are over $(a, b) \in \mathcal{L}$ and $\left(a^{\prime}, b^{\prime}\right) \in \mathcal{L}$, while $\Sigma^{3}$ is over $|y(3)|<\nu$ and $|y(4)|<v$.

Now consider a typical summand of (7.18),

$$
C_{7} \equiv \iint K_{n}(\mu-\eta) K_{n}\left(\mu^{\prime}-\eta^{\prime}\right) f_{a, b}\left(\lambda^{\prime}+2 \pi k\right) f_{a^{\prime}, b^{\prime}}\left(-\mu^{\prime}+2 \pi k^{\prime}\right)
$$

$$
\times \operatorname{sinc}(a, y(1)) \operatorname{sinc}\left(a^{\prime}, y(2)\right) d \mu d \mu^{\prime} .
$$

For fixed $k, k^{\prime},(a, b) \in \mathcal{L},\left(a^{\prime}, b^{\prime}\right) \in \mathscr{L}$, we evaluate $C_{7}$ in the following cases:

(i) If $a \alpha^{\prime} \neq a^{\prime} \alpha$, because of the form of $y(1)$ and $y(2)$, we introduce the change of variables

$$
a \alpha^{\prime} \mu^{\prime}-\alpha \mu=x, \quad a^{\prime} \mu^{\prime}-\mu=x^{\prime}
$$

with the Jacobian

$$
J=\left|\begin{array}{rr}
a \alpha^{\prime} & -\alpha \\
a^{\prime} & -1
\end{array}\right|=-a \alpha^{\prime}+a^{\prime} \alpha \neq 0
$$

and

$$
\mu=J^{-1}\left(a \alpha^{\prime} x^{\prime}-a^{\prime} x\right), \quad \mu^{\prime}=J^{-1}\left(\alpha x^{\prime}-x\right) .
$$


We have

$$
\begin{aligned}
C_{7}=\iint_{\Omega} & K_{n}\left(J^{-1}\left(a \alpha^{\prime} x^{\prime}-a^{\prime} x\right)-\eta\right) K_{n}\left(J^{-1}\left(\alpha x^{\prime}-x\right)-\eta^{\prime}\right) \\
& \times f_{a, b}\left(\alpha^{\prime} J^{-1}\left(\alpha x^{\prime}-x\right)+w^{\prime}+2 \pi k\right) f_{a^{\prime}, b^{\prime}}\left(-J^{-1}\left(\alpha x^{\prime}-x\right)+2 \pi k^{\prime}\right) \\
& \times \operatorname{sinc}\left(a,\left(2 \pi k a+x+a w^{\prime}+b-w\right) \bmod 2 \pi\right) \\
& \times \operatorname{sinc}\left(a^{\prime},\left(2 \pi k^{\prime} a^{\prime}-x^{\prime}+b^{\prime}\right) \bmod 2 \pi\right) J^{-1} d x d x^{\prime},
\end{aligned}
$$

where $\Omega$ is a bounded region. Using Assumption 3 and the fact that $K$ is bounded, there is a constant $c$ such that

$$
\begin{aligned}
\left|C_{7}\right| \leq & \frac{c}{b_{n}^{2}} g\left(x_{1}+2 \pi k\right) g\left(x_{2}+2 \pi k^{\prime}\right) \\
& \times \int_{\Omega} \mid \operatorname{sinc}\left(a^{\prime},\left(2 \pi k a+x+a w^{\prime}+b-w\right) \bmod 2 \pi\right) \\
& \quad \times \operatorname{sinc}\left(a^{\prime},\left(2 \pi k^{\prime} a^{\prime}-x^{\prime}+b^{\prime}\right) \bmod 2 \pi\right) \mid d x d x^{\prime} \\
= & O\left(\frac{\log ^{2} n}{n^{2} b_{n}^{2}}\right) g\left(x_{1}+2 \pi k\right) g\left(x_{2}+2 \pi k^{\prime}\right),
\end{aligned}
$$

with $x_{1}, x_{2}$ some fixed values. Using Assumptions 1 and 2, the contribution to $C_{5}$ is shown to be $O\left(\log ^{2} n /\left(n^{2} b_{n}^{2}\right)\right)$.

(ii) If $a \alpha^{\prime}=a^{\prime} \alpha$, then, by a change of variable,

$$
\begin{gathered}
x=a \alpha^{\prime} \mu^{\prime}-\alpha \mu, \quad \mu=\frac{1}{\alpha}\left(a \alpha^{\prime} \mu^{\prime}-x\right)=a^{\prime} \mu^{\prime}-\frac{1}{\alpha} x \\
C_{7}=-\frac{1}{\alpha} \int_{-\pi^{+}}^{\pi^{+}} \int_{A} K_{n}\left(-\frac{1}{\alpha} x+a^{\prime} \mu^{\prime}-\eta\right) K_{n}\left(\mu^{\prime}-\eta^{\prime}\right) f_{a, b}\left(\alpha^{\prime} \mu^{\prime}+w^{\prime}+2 \pi k\right) \\
\times f_{a^{\prime}, b^{\prime}}\left(-\mu^{\prime}+2 \pi k^{\prime}\right) \operatorname{sinc}\left(a,\left(x+y_{k, 1}\right)_{2 \pi}\right) \\
\times \operatorname{sinc}\left(a^{\prime},\left(\frac{-1}{\alpha}\left(x-\alpha y_{k^{\prime}, 2}\right)\right)_{2 \pi}\right) I_{A\left(\mu^{\prime}\right)}(x) d x d \mu^{\prime}
\end{gathered}
$$

where

$$
y_{k, 1}=2 \pi k a+a w^{\prime}+b-w, \quad y_{k^{\prime}, 2}=2 \pi k^{\prime} a^{\prime}+b^{\prime},
$$

with

$$
A=\left[-\left(a \alpha^{\prime}+\alpha\right) \pi^{+},\left(a \alpha^{\prime}+\alpha\right) \pi^{+}\right], \quad A\left(\mu^{\prime}\right)=\left[a \alpha^{\prime} \mu^{\prime}-\alpha \pi^{+}, a \alpha^{\prime} \mu^{\prime}+\alpha \pi^{+}\right] .
$$

Let $x^{\prime}=\left(\mu^{\prime}-\eta^{\prime}\right) b_{n}^{-1}, \mu^{\prime}=\eta^{\prime}+b_{n} x^{\prime}$. Then

$$
\begin{array}{rl}
C_{7}=-\frac{1}{\alpha b_{n}} \int_{A_{1}} \int_{A} & K\left(a^{\prime} x^{\prime}+\frac{1}{b_{n}}\left(a^{\prime} \eta^{\prime}-\eta-\frac{1}{\alpha} x\right)\right) K\left(x^{\prime}\right) \\
& \times f_{a, b}\left(\alpha^{\prime} \eta^{\prime}+\alpha^{\prime} b_{n} x^{\prime}+w^{\prime}+2 \pi k\right) f_{a^{\prime}, b^{\prime}}\left(-\eta^{\prime}-b_{n} x^{\prime}+2 \pi k^{\prime}\right)
\end{array}
$$




$$
\begin{aligned}
& \times \operatorname{sinc}\left(a,\left(x+y_{k, 1}\right)_{2 \pi}\right) \operatorname{sinc}\left(a^{\prime},-\left(\frac{1}{\alpha}\left(x-\alpha y_{k^{\prime}, 2}\right)\right)_{2 \pi}\right) \\
& \times I_{A\left(\eta^{\prime}+b_{n} x^{\prime}\right)}(x) d x d x^{\prime}
\end{aligned}
$$

with $A_{1}=\left[\left(-\pi^{+}-\eta^{\prime}\right) b_{n}^{-1},\left(\pi^{+}-\eta^{\prime}\right) b_{n}^{-1}\right]$. Let $z=x+y_{k, 1}, x=z-y_{k, 1}$ [we note that since $\left(x+y_{k, 1}\right)_{2 \pi}=\left(x+\left(y_{k, 1}\right)_{2 \pi}\right)_{2 \pi}$ we can replace $y_{k, 1}$ by $\left(y_{k, 1}\right)_{2 \pi}$ in the following]

$$
\begin{aligned}
C_{7}=\frac{-1}{\alpha b_{n}} \int_{A_{1}} \int_{A^{\prime}} & K\left(a^{\prime} x^{\prime}+\frac{1}{b_{n}}\left(a^{\prime} \eta^{\prime}-\eta+\frac{1}{\alpha} y_{k, 1}-\frac{1}{\alpha} z\right)\right) K\left(x^{\prime}\right) \\
& \times f_{a, b}\left(\alpha^{\prime} \eta^{\prime}+w^{\prime}+2 \pi k+b_{n} \alpha^{\prime} x^{\prime}\right) f_{a^{\prime}, b^{\prime}}\left(-\eta^{\prime}+2 \pi k^{\prime}-b_{n} x^{\prime}\right) \\
& \times \operatorname{sinc}\left(a, z_{2 \pi}\right) \operatorname{sinc}\left(a^{\prime},-\left(\frac{1}{\alpha}\left(z-y_{k, 1}-\alpha y_{k^{\prime}, 2}\right)\right)_{2 \pi}\right) \\
& \times I_{A\left(\eta^{\prime}+b_{n} x^{\prime}\right)}\left(z-y_{k, 1}\right) d z d x^{\prime}
\end{aligned}
$$

where $A^{\prime}=A+y_{k, 1}$.

Let $\mathcal{L}^{\prime}=\left\{l \mid 2 \pi l \in A^{\prime}, l\right.$ integers $\}$ which is a finite set.

For each $l \in \mathcal{L}^{\prime}$, let $A^{l}=[-\pi+2 \pi l, \pi+2 \pi l] \cap A^{\prime}$. Then

$$
\begin{array}{rl}
C_{7}=\frac{-1}{\alpha b_{n}} \sum_{l \in \mathcal{L}^{\prime}} \int_{A_{1}} \int_{A^{l}} & K\left(a^{\prime} x^{\prime}+\frac{1}{b_{n}}\left(a^{\prime} \eta^{\prime}-\eta+\frac{1}{\alpha} y_{k, 1}-\frac{1}{\alpha} z\right)\right) K\left(x^{\prime}\right) \\
& \times f_{a, b}\left(\alpha^{\prime} \eta^{\prime}+\alpha^{\prime} b_{n} x^{\prime}+w^{\prime}+2 \pi k\right) f_{a^{\prime}, b^{\prime}}\left(-\eta^{\prime}-b_{n} x^{\prime}+2 \pi k^{\prime}\right) \\
& \times \operatorname{sinc}\left(a, z_{2 \pi}\right) \operatorname{sinc}\left(a^{\prime},-\left(\frac{1}{\alpha}\left(z-y_{k, 1}-\alpha y_{k^{\prime}, 2}\right)\right)_{2 \pi}\right) \\
& \times I_{A\left(\eta^{\prime}+b_{n} x^{\prime}\right)}\left(z-y_{k, 1}\right) d z d x^{\prime} .
\end{array}
$$

Letting $z^{\prime}=z-2 \pi l$,

$$
C_{7}=\sum_{l \in \mathcal{L}^{\prime}} \frac{-1}{\alpha b_{n}} G_{l}
$$

with

$$
\begin{array}{rl}
G_{l}=\int_{A_{1}} \int_{A^{l}-2 \pi l} & K\left(a^{\prime} x^{\prime}+\frac{1}{b_{n}}\left(a^{\prime} \eta^{\prime}-\eta+\frac{1}{\alpha} y_{k, 1}-\frac{1}{\alpha} 2 \pi l-\frac{1}{\alpha} z^{\prime}\right)\right) K\left(x^{\prime}\right) \\
& \times f_{a, b}\left(\alpha^{\prime} \eta^{\prime}+w^{\prime}+2 \pi k+b_{n} \alpha^{\prime} x^{\prime}\right) f_{a^{\prime}, b^{\prime}}\left(-\eta^{\prime}+2 \pi k^{\prime}-b_{n} x^{\prime}\right) \\
& \times \operatorname{sinc}\left(a, z^{\prime}\right) \operatorname{sinc}\left(a^{\prime},-\left(\frac{1}{\alpha}\left(z^{\prime}+2 \pi l-y_{k, 1}-\alpha y_{k^{\prime}, 2}\right)\right)_{2 \pi}\right) \\
& \times I_{A\left(\eta^{\prime}+b_{n} x^{\prime}\right)}\left(z^{\prime}+2 \pi l-y_{k, 1}\right) d z^{\prime} d x^{\prime} .
\end{array}
$$


We note that $A_{c}^{l} \equiv A^{l}-2 \pi l$ is centered on 0 with length at most $2 \pi$. For each $l \in \mathscr{L}^{\prime}$, rewriting this last expression with $q_{l} \equiv a^{\prime} \eta^{\prime}-\eta+\frac{1}{\alpha} y_{k, 1}-\frac{1}{\alpha} 2 \pi l \neq 0$ and $J\left(l, z^{\prime}, x^{\prime}\right)=I_{A\left(\eta^{\prime}+b_{n} x^{\prime}\right)}\left(z^{\prime}+2 \pi l-y_{k, 1}\right)$, we have

$$
\begin{array}{rl}
G_{l}=\int_{A_{1}} \int_{A_{c}^{l}} & K\left(a^{\prime} x^{\prime}+\frac{1}{b_{n}}\left(q_{l}-\frac{1}{\alpha} z^{\prime}\right)\right) K\left(x^{\prime}\right) \\
& \times f_{a, b}\left(\alpha^{\prime} \eta^{\prime}+w^{\prime}+2 \pi k+b_{n} \alpha^{\prime} x^{\prime}\right) f_{a^{\prime}, b^{\prime}}\left(-\eta^{\prime}+2 \pi k^{\prime}-b_{n} x^{\prime}\right) \\
& \times \operatorname{sinc}\left(a, z^{\prime}\right) \operatorname{sinc}\left(a^{\prime},-\left(\frac{1}{\alpha}\left(z^{\prime}+2 \pi l-y_{k, 1}-\alpha y_{k^{\prime}, 2}\right)\right)_{2 \pi}\right) \\
& \times J\left(l, z^{\prime}, x^{\prime}\right) d z^{\prime} d x^{\prime} .
\end{array}
$$

Let $e_{n}$ be a sequence such that

$$
e_{n}=o\left(b_{n}\right), \quad n^{-1}=o\left(e_{n}\right), \quad n^{-1}=o\left(b_{n}\right), \quad c_{0}, c_{1} \text { constants. }
$$

Then there exist constants $c_{0}, x_{1}$ and $x_{2}$ such that

$$
\left|G_{l}\right| \leq c_{0} g\left(x_{1}+2 \pi k\right) g\left(x_{2}+2 \pi k^{\prime}\right)
$$

$$
\times \int_{A_{1}}\left[\int_{\left|z^{\prime}\right| \leq c_{1} e_{n}}+\int_{\pi \geq\left|z^{\prime}\right|>c_{1} e_{n}}\right]\left|G\left(z^{\prime}, x^{\prime}\right)\right| d z^{\prime},
$$

where $G\left(z^{\prime}, x^{\prime}\right)=G^{\prime}\left(z^{\prime}, x^{\prime}\right) G^{\prime \prime}\left(z^{\prime}\right), G^{\prime}\left(z^{\prime}, x^{\prime}\right)=K\left(a^{\prime} x^{\prime}+\left(1 / b_{n}\right)\left(q_{l}-(1 / \alpha) z^{\prime}\right)\right)$ $K\left(x^{\prime}\right)$,

$$
G^{\prime \prime}\left(z^{\prime}\right)=\operatorname{sinc}\left(a, z^{\prime}\right) \operatorname{sinc}\left(a^{\prime},-\left(\frac{1}{\alpha}\left(z^{\prime}+2 \pi l-y_{k, 1}-\alpha y_{k^{\prime}, 2}\right)\right)_{2 \pi}\right) .
$$

We see that the first double integral in (7.23) is $\int_{\left|z^{\prime}\right| \leq c_{1} e_{n}}\left(\int_{A_{1}}\left|G^{\prime}\left(z^{\prime}, x^{\prime}\right)\right| d x^{\prime}\right)$ $\times\left|G^{\prime \prime}\left(z^{\prime}\right)\right| d z^{\prime}$ and $\int_{A_{1}}\left|G^{\prime}\left(z^{\prime}, x^{\prime}\right)\right| d x^{\prime}=o(1)$ uniformly in $z^{\prime}$ since $e_{n}=o\left(b_{n}\right)$ and $\left(1 / b_{n}\right)\left(q_{l}-\frac{1}{\alpha} z^{\prime}\right) \rightarrow \infty$. (We can also use the fact that $x^{\prime}$ has finite range or $K$ has finite support.) Note that $\int_{\left|z^{\prime}\right| \leq c e_{n}}\left|G^{\prime \prime}\left(z^{\prime}\right)\right| d z^{\prime}=O\left(\frac{1}{n}\right)$ by the Schwarz inequality. The second double integral in (7.23) is $\int_{c_{1} e_{n} \leq\left|z^{\prime}\right| \leq \pi}\left(\left|G^{\prime \prime}\left(z^{\prime}\right)\right| \int_{A_{1}}\left|G^{\prime}\left(x^{\prime}, x^{\prime}\right)\right| d x^{\prime}\right)$ $\times d z^{\prime}$. But

$$
\int_{A_{1}}\left|G^{\prime}\left(z^{\prime}, x^{\prime}\right)\right| d x^{\prime} \leq\left|a^{\prime}\right|^{-1 / 2} \int K^{2}(y) d y
$$

by the Schwarz inequality and

$$
\begin{aligned}
& \int_{c_{1} e_{n} \leq\left|z^{\prime}\right| \leq \pi}\left|G^{\prime \prime}\left(z^{\prime}\right)\right| d z^{\prime} \\
& =O\left(\frac{1}{n^{2}}\right) \int_{c_{1} e_{n} \leq\left|z^{\prime}\right| \leq \pi} \frac{\sin \left(((n+1) / 2) z^{\prime} l(a)\right)}{z^{\prime}}
\end{aligned}
$$




$$
\begin{aligned}
& \times \frac{\sin \left\{\left((n+1 / 2)\left[-(1 / \alpha)\left(z^{\prime}+2 \pi l-y_{k, 1}-\alpha y_{k^{\prime}, 2}\right)\right]_{2 \pi} l\left(a^{\prime}\right)\right\}\right.}{\left(-(1 / \alpha)\left(z^{\prime}+2 \pi l-y_{k, 1}-\alpha y_{k^{\prime}, 2}\right)\right)_{2 \pi}} d z^{\prime} \\
= & o\left(\frac{1}{n}\right) .
\end{aligned}
$$

Therefore, when $q_{l} \neq 0,\left|G_{l}\right|=o(1 / n) g\left(x_{1}+2 \pi k\right) g\left(x_{2}+2 \pi k^{\prime}\right)$. In this case, by Assumptions 1 and 2, the contribution from $C_{7}$ to $C_{5}$ is shown to be $o\left(1 / n b_{n}\right)$.

If $q_{l}=a^{\prime} \eta^{\prime}-\eta+\frac{1}{\alpha} y_{k, 1}-\frac{1}{\alpha} 2 \pi l=0$,

$$
\begin{array}{rl}
G_{l}=\int_{A_{1}} \int_{A_{c}^{l}} & K\left(a^{\prime} x^{\prime}-\frac{1}{\alpha} z^{\prime} / b_{n}\right) K\left(x^{\prime}\right) \\
& \times f_{a, b}\left(\alpha^{\prime} \eta^{\prime}+w^{\prime}+2 \pi k+b_{n} \alpha^{\prime} x^{\prime}\right) f_{a^{\prime}, b^{\prime}}\left(-\eta^{\prime}+2 \pi k^{\prime}-b_{n} x^{\prime}\right) \\
& \times J\left(l, z^{\prime}, x^{\prime}\right) G^{\prime \prime}\left(z^{\prime}\right) d z^{\prime} d x^{\prime}
\end{array}
$$$$
=\left[\int_{A_{1}} \int_{\left\{\left|z^{\prime}\right| \leq c_{1} e_{n}\right\} \cap A_{c}^{l}}+\int_{A_{1}} \int_{\left\{\left|z^{\prime}\right| \geq c_{1} e_{n}\right\} \cap A_{c}^{l}}\right] G^{\prime \prime \prime}\left(z^{\prime}, x^{\prime}\right) G^{\prime \prime}\left(z^{\prime}\right)
$$$$
\times J\left(l, z^{\prime}, x^{\prime}\right) d z^{\prime} d x^{\prime},
$$

where

$$
\begin{aligned}
G^{\prime \prime \prime}\left(z^{\prime}, x^{\prime}\right)= & K\left(a^{\prime} x^{\prime}-\frac{1}{\alpha} z^{\prime} / b_{n}\right) K\left(x^{\prime}\right) f_{a, b}\left(\alpha^{\prime} \eta^{\prime}+w^{\prime}+2 \pi k+b_{n} \alpha^{\prime} x^{\prime}\right) \\
& \times f_{a^{\prime}, b^{\prime}}\left(-\eta^{\prime}+2 \pi k^{\prime}-b_{n} x^{\prime}\right) .
\end{aligned}
$$

Using the same argument as before, we see that the second double integral in (7.24) is $o(1 / n) g\left(x_{1}+2 \pi k\right) g\left(x_{2}+2 \pi k^{\prime}\right)$. Its total contribution to $C_{5}$ is $o\left(1 / n b_{n}\right)$. The first double integral can be approximated by $G_{l, 1}$ given below using the fact that $\left|z^{\prime}\right| \leq c_{1} e_{n}, e_{n}=o\left(b_{n}\right)$ and Lemma 7.3 (at the end of this section) with $y=x^{\prime}$ and $z=z^{\prime}$, noting that $G^{\prime \prime}$ and $J$ are bounded.

$$
\begin{aligned}
G_{l, 1} \equiv \int_{\left\{\left|z^{\prime}\right| \leq c_{1} e_{n}\right\} \cap A_{c}^{l}} \int_{A_{1}} K\left(a^{\prime} x^{\prime}\right) K\left(x^{\prime}\right) f_{a, b}\left(a^{\prime} \eta^{\prime}+w^{\prime}+2 \pi k+b_{n} \alpha^{\prime} x^{\prime}\right) \\
\quad \times f_{a^{\prime}, b^{\prime}}\left(-\eta^{\prime}+2 \pi k^{\prime}-b_{n} x^{\prime}\right) I_{A\left(\eta^{\prime}+b_{n} x^{\prime}\right)}\left(z^{\prime}+2 \pi l-y_{k, 1}\right) G^{\prime \prime}\left(z^{\prime}\right) d x^{\prime} d z^{\prime} .
\end{aligned}
$$

The weight function $K$ has finite support $A_{K}$ and by Assumption 2 we have

$$
\begin{aligned}
& G_{l, 1}= \int_{A_{K}} K\left(a^{\prime} x^{\prime}\right) K\left(x^{\prime}\right) d x^{\prime} f_{a, b}\left(a^{\prime} \eta^{\prime}+w^{\prime}+2 \pi k\right) f_{a^{\prime}, b^{\prime}}\left(-\eta^{\prime}+2 \pi k^{\prime}\right) \\
& \times \int_{\left\{\left|z^{\prime}\right| \leq c_{1} e_{n}\right\} \cap A_{c}^{l}} \operatorname{sinc}\left(a, z^{\prime}\right) \operatorname{sinc}\left(a^{\prime},-\left(\frac{1}{\alpha}\left(z^{\prime}+2 \pi l-y_{k, 1}-\alpha y_{k^{\prime}, 2}\right)\right)_{2 \pi}\right) \\
& \times I_{A\left(\eta^{\prime}+b_{n} x^{\prime}\right)}\left(z^{\prime}+2 \pi l-y_{k, 1}\right) d z^{\prime} d x^{\prime}
\end{aligned}
$$




$$
\begin{aligned}
& +O\left(b_{n}\right) g\left(a^{\prime} \eta^{\prime *}+w^{\prime}+2 \pi k\right) g\left(-\eta^{\prime *}+2 \pi k^{\prime}\right) \int_{\left\{\left|z^{\prime}\right| \leq c_{1} e_{n}\right\} \cap A_{c}^{l}}\left|G^{\prime \prime}\left(z^{\prime}\right)\right| d z^{\prime} \\
\equiv & G_{l, 1}^{\prime}+G_{l, 1}^{\prime \prime} .
\end{aligned}
$$

It is clear that $\left|G_{l, 1}^{\prime \prime}\right| \leq O\left(b_{n}\right) g\left(a^{\prime} \eta^{\prime *}+w^{\prime}+2 \pi k\right) g\left(-\eta^{\prime *}+2 \pi k^{\prime}\right) O\left(\frac{1}{n}\right)$ and its total contribution to $C_{5}$ is $O\left(\frac{1}{n}\right)$. To evaluate $G_{l, 1}^{\prime}$, let

$$
A_{3} \equiv\left[a \alpha^{\prime} \eta^{\prime}-\alpha \pi^{+}-2 \pi l+y_{k, 1}, a \alpha^{\prime} \eta^{\prime}+\alpha \pi^{+}-2 \pi l+y_{k, 1}\right] .
$$

Note that

$$
I_{A\left(\eta^{\prime}+b_{n} x^{\prime}\right)}\left(z^{\prime}+2 \pi l-y_{k, 1}\right)=I_{A_{3}^{\prime}}\left(z^{\prime}\right)
$$

with

$A_{3}^{\prime}=\left[a \alpha^{\prime}\left(\eta^{\prime}+b_{n} x^{\prime}\right)-\alpha \pi^{+}-2 \pi l+y_{k, 1}, a \alpha^{\prime}\left(\eta^{\prime}+b_{n} x^{\prime}\right)+\alpha \pi^{+}-2 \pi l+y_{k, 2}\right]$.

The condition $q_{l}=0$ means $a^{\prime} \alpha \eta^{\prime}-\alpha \eta+y_{k, 1}-2 \pi l=0$. This means $a \alpha^{\prime} \eta^{\prime}-$ $\alpha \eta+y_{k, 1}-2 \pi l=0$ by the condition $a^{\prime} \alpha=a \alpha^{\prime}$. Now $\eta \in[-\pi, \pi], \pi^{+}>\pi, x^{\prime}$ is bounded. Therefore $0 \in A_{3}^{0}$, the interior of $A_{3}$, and then $I_{A_{3}}\left(z^{\prime}\right)=1$ when $\left|z^{\prime}\right| \leq c_{1} e_{n}$ for large $n$. Also $q_{l}=0$ implies $0 \in A_{c}^{l}$, so, for large $n$, the inner integral of $G_{l, 1}^{\prime}$ is

$$
G^{4} \equiv \int_{\left|z^{\prime}\right| \leq c_{1} e_{n}} \operatorname{sinc}\left(a, z^{\prime}\right) \operatorname{sinc}\left(a^{\prime},-\left(\frac{1}{\alpha}\left(z^{\prime}+2 \pi l-y_{k, 1}-\alpha y_{k^{\prime}, 2}\right)\right)_{2 \pi}\right) d z^{\prime} .
$$

If $q_{l}^{\prime} \equiv\left(\frac{1}{\alpha} y_{k, 1}+y_{k^{\prime}, 2}-\frac{1}{\alpha} 2 \pi l\right)_{2 \pi} \neq 0$, then $G^{4}=o\left(\frac{1}{n}\right)$. If $q_{l}^{\prime}=0$, then, for large $n$,

$$
\begin{aligned}
G^{4} & =\int_{\left|z^{\prime}\right| \leq c_{1} e_{n}} \operatorname{sinc}\left(a, z^{\prime}\right) \operatorname{sinc}\left(a^{\prime},-\frac{1}{\alpha} z^{\prime}\right) d z^{\prime} \\
& =-\alpha \int_{|x| \leq c_{1} e_{n} / \alpha} \operatorname{sinc}(a,-\alpha x) \operatorname{sinc}\left(a^{\prime}, x\right) d x .
\end{aligned}
$$

Following an argument like that leading to (7.7)-(7.12) and using the Parseval identity, we have

$$
\int_{|x| \leq c_{1} e_{n} / \alpha} \operatorname{sinc}(a,-\alpha x) \operatorname{sinc}\left(a^{\prime}, x\right) d x=\frac{2 \pi}{n+1} l\left(\alpha, \alpha^{\prime}, a, a^{\prime}\right)+o\left(\frac{1}{n}\right)
$$

with

$$
l\left(\alpha, \alpha^{\prime}, a, a^{\prime}\right)=\min \left(\alpha^{\prime} \frac{\min (a, 1)}{\min \left(a^{\prime}, 1\right)}, 1\right) \frac{\min \left(a^{\prime}, 1\right)}{a \alpha^{\prime}} .
$$

In this case,

$$
\begin{aligned}
G_{l, 1}= & f_{a, b}\left(a^{\prime} \eta^{\prime}+w^{\prime}+2 \pi k\right) f_{a^{\prime}, b^{\prime}}\left(-\eta^{\prime}+2 \pi k^{\prime}\right) \int_{A_{K}} K\left(a^{\prime} x^{\prime}\right) K\left(x^{\prime}\right) d x^{\prime} \\
& \times\left[\frac{-\alpha 2 \pi}{n+1} l\left(\alpha, \alpha^{\prime}, a, a^{\prime}\right)+o\left(\frac{1}{n}\right)\right] .
\end{aligned}
$$


In summary, under the assumption $a \alpha^{\prime}=a^{\prime} \alpha$,

$$
\begin{aligned}
C_{7}= & \frac{1}{b_{n}} \sum_{l \in \mathscr{L}^{\prime \prime}} f_{a, b}\left(a^{\prime} \eta^{\prime}+w^{\prime}+2 \pi k\right) f_{a^{\prime}, b^{\prime}}\left(-\eta^{\prime}+2 \pi k^{\prime}\right) \\
& \times\left[\int K\left(a^{\prime} x^{\prime}\right) K\left(x^{\prime}\right) d x^{\prime} \frac{2 \pi}{n+1} l\left(\alpha, \alpha^{\prime}, a, a^{\prime}\right)+o\left(\frac{1}{n}\right)\right],
\end{aligned}
$$

where, for each $k, k^{\prime}, a, b, a^{\prime}, b^{\prime}, \mathcal{L}^{\prime \prime}=\mathscr{L}^{\prime} \cap \mathscr{L}_{1}^{\prime} \cap \mathscr{L}_{2}^{\prime}$ with

$$
\begin{gathered}
\mathcal{L}^{\prime}=\left\{l \mid 2 \pi l \in A^{\prime} \equiv\left[-\left(a \alpha^{\prime}+\alpha\right) \pi^{+},(a \alpha+\alpha) \pi^{+}\right]+y_{k, 1}\right\}, \\
y_{k, 1}=2 \pi k a+a w^{\prime}+b-w, \\
\mathcal{L}_{1}^{\prime}=\left\{l \mid q_{l} \equiv a^{\prime} \eta^{\prime}-\eta+\frac{1}{\alpha}\left(y_{k, 1}-2 \pi l\right)=0\right\}, \\
\mathcal{L}_{2}^{\prime}=\left\{l \mid q_{l}^{\prime}=\left(\frac{1}{\alpha}\left(y_{k, 1}-2 \pi l\right)+y_{k^{\prime}, 2}\right)_{2 \pi}=0\right\}
\end{gathered}
$$

and

$$
y_{k^{\prime}, 2}=2 \pi k^{\prime} a^{\prime}+b^{\prime} .
$$

However, we note that if there is an $l \in \mathcal{L}_{1}^{\prime}$ then $l \in \mathcal{L}^{\prime}$ and the condition $q_{l}=0$ is

$$
\begin{aligned}
0 & =\alpha a^{\prime} \eta^{\prime}-\alpha \eta+2 \pi k a+a w^{\prime}+b-w-2 \pi l \\
& =2 \pi k a+b+a\left(\alpha^{\prime} \eta^{\prime}+w^{\prime}\right)-(\alpha \eta+w)-2 \pi l
\end{aligned}
$$

by the condition $a \alpha^{\prime}=\alpha a^{\prime}$. This is the same as $y\left(k, a, b ; \xi, \xi^{\prime}\right)=0 \bmod 2 \pi$. Substituting $q_{l}=0$ into $q_{l}^{\prime}=0$ in $\mathcal{L}_{2}^{\prime}$, we have $\left(-a^{\prime} \eta^{\prime}+\eta+y_{k^{\prime}, 2}\right)_{2 \pi}=0$. This is the same as $y\left(k^{\prime}, a^{\prime}, b^{\prime},-\eta,-\eta^{\prime}\right)=0 \bmod 2 \pi$. Therefore the sum over $l \in \mathcal{L}^{\prime \prime}$ in $(7.25)$ can be replaced by the restriction

$$
\delta_{a \alpha^{\prime}-a^{\prime} \alpha, 0} \delta_{\left(y\left(k ; a, b ; \xi, \xi^{\prime}\right)\right), 0} \delta_{\left(y\left(k^{\prime} ; a^{\prime}, b^{\prime} ;-\eta,-\eta^{\prime}\right)\right), 0} .
$$

Substituting the modified (7.25) into (7.20) and (7.18), we have $C_{5}$ in (7.17) and the first term in (5.8). By a similar argument for $C_{6}$ in (7.19), we obtain the second term in (5.8). This completes the proof.

LEMMA 7.3. If $K(x) \in L_{\infty} \cap L_{1}$ and

$$
K(x) \rightarrow 0 \text { as }|x| \rightarrow \infty,
$$

then, for $e \neq 0$,

$$
\int(K(e y+z)-K(e y)) K(y) g(y, z) d y \rightarrow 0 \quad \text { as } z \rightarrow 0
$$

for a bounded function $g$. 
Proof. Let $|g| \leq c$. Then

$$
\begin{aligned}
& \int(K(e y+z)-K(e y)) g(y, z) K(y) d y \\
& \quad=\left[\int_{|y| \geq L}+\int_{|y| \leq L}\right](K(y) g(y, z)(K(e y+z)-K(e y))) d y .
\end{aligned}
$$

Given any $\varepsilon>0$, since $K(x) \in L_{1}$ and $K(x) \rightarrow 0$ as $|x| \rightarrow \infty$ there exists an $L>0$ such that

$$
\left|\int_{|y| \geq L} g(y, z) K(y)(K(e y+z)-K(e y)) d y\right|<\varepsilon / 2 .
$$

By Lusin's theorem, there exists a continuous function $K_{c, \varepsilon}(y)$ such that

$$
\int_{|y| \leq L}\left|K(e y)-K_{c, \varepsilon}(e y)\right|^{2} d y \leq \frac{\varepsilon^{2}}{36 M^{2}},
$$

where $M^{2}=c^{2} \int K^{2}(y) d y$. Then

$$
\begin{aligned}
& \left|\int_{|y| \leq L} g(y, z) K(y)(K(e y)-K(e y+z))\right| d y \\
& \leq\left[\int_{|y| \leq L} g^{2}(y, z) K^{2}(y) d y \int_{|y| \leq L}(K(e y)-K(e y+z))^{2} d y\right]^{1 / 2} \\
& =M\left\{\int _ { | y | \leq L } \left[K(e y)-K_{c, \varepsilon}(e y)+K_{c, \varepsilon}(e y)-K_{c, \varepsilon}(e y+z)\right.\right. \\
& \left.\left.\quad+K_{c, \varepsilon}(e y+z)-K(e y+z)\right]^{2} d y\right\}^{1 / 2} \\
& \leq \sqrt{3} M\left\{\int_{|y| \leq L}\left[K(e y)-K_{c, \varepsilon}(e y)\right]^{2} d y\right. \\
& \quad+\int_{|y| \leq L}\left[K_{c, \varepsilon}(e y)-K_{c, \varepsilon}(e y+z)\right]^{2} d y \\
& \left.\quad+\int_{|y| \leq L}\left[K_{c, \varepsilon}(e y+z)-K(e y+z)\right]^{2}\right\}^{1 / 2} \\
& \leq \sqrt{3} M\left[\frac{\varepsilon^{2}}{36 M^{2}}+\frac{\varepsilon^{2}}{36 M^{2}}+\frac{\varepsilon^{2}}{36 M^{2}}\right]^{1 / 2}=\frac{\varepsilon}{2}
\end{aligned}
$$

since

$$
\int_{|y| \leq L}\left(K_{c, \varepsilon}(e y)-K_{c, \varepsilon}(e y+z)\right)^{2} d y \leq \frac{\varepsilon^{2}}{36 M^{2}}
$$

for $z$ sufficiently small by the continuity of $K_{c, \varepsilon}(u)$ for $|u| \leq 2 L$. 
Therefore, for any $\varepsilon>0$, one can choose $z$ sufficiently small such that

$$
\left|\int g(y, z) K(y)(K(e y)-K(e y+z)) d y\right| \leq \varepsilon
$$

and the lemma is proved.

\section{REFERENCES}

Alekseev, V. G. (1988). Estimating the spectral densities of a Gaussian periodically correlated stochastic process. Problems Inform. Transmission 24 109-115.

Allen, J. and HobBS, S. (1992). Detecting target motion by frequency-plane smoothing. In Asilomar Conference on Signals, Systems and Computers (A. Singh, ed.) 1042-1047.

BoHr, H. A. (1951). Almost Periodic Functions. Chelsea, New York.

CHIU, S.-T. (1986). Statistical estimation of the parameters of a moving source from array data. Ann. Statist. 14 559-578.

DAHLhaus, R. (1997). Fitting time series models to nonstationary processes. Ann. Statist. 25 1-37.

DANDAWATE, A. V. and GIANNAKIS, G. B. (1994). Nonparametric polyspectral estimators for $k$ thorder (almost) cyclostationary processes. IEEE Trans. Inform. Theory 40 67-84.

Daniell, P. J. (1946). Discussion of paper by M. S. Bartlett. J. Roy. Statist. Soc. (Suppl.) 8 88-90.

DEHAY, D. and HuRD, H. L. (1993). Representation and estimation for periodically and almost periodically correlated random processes. In Cyclostationarity in Communications and Signal Processing (W. A. Gardner, ed.). IEEE Press, New York.

FERguson, B. G. (1999). Time-delay estimation techniques applied to the acoustic detection of jet aircraft transits. J. Acoust. Soc. Amer. 106 255-264.

GARDNER, W. A. (1991). Exploitation of spectral redundancy in cyclostationary signals. IEEE Signal Processing Magazine 8 14-36.

GARDNER, W. A. (1994). Cyclostationarity in Communications and Signal Processing. IEEE Press, New York.

GERR, N. and Allen, J. (1994a). The generalized spectrum and spectral coherence of a harmonizable time series. Digital Signal Processing 4 222-238.

Gerr, N. and Allen, J. (1994b). Time-delay estimation for harmonizable signals. Digital Signal Processing 4 49-62.

GLADYSHEV, E. G. (1963). Periodically and almost periodically correlated random processes with continuous time parameters. Theory Probab. Appl. 8 173-177.

HURD, H. (1989). Nonparametric time series analysis for periodically correlated processes. IEEE Trans. Inform. Theory 35 350-359.

Hurd, H. and GERR, N. (1991). Graphical methods for determining the presence of periodic correlation. J. Time Ser. Anal. 12 337-350.

JiN, Q., Wong, K. M. and LuO, Z. Q. (1995). The estimation of time delay and Doppler stretch of wideband signals. IEEE Trans. Signal Processing 43 904-916.

LESKOW, J. and WERON, A. (1992). Ergodic behavior and estimation for periodically correlated processes. Statist. Probab. Lett. 15 299-304.

LII, K. S. and RosenblatT, M. (1998). Line spectral analysis for harmonizable processes. Proc. Nat. Acad. Sci. USA 95 4800-4803.

LoÈve, M. (1963). Probability Theory, 3rd ed. Van Nostrand, Princeton, NJ.

MALlat, S., PAPANICOLAOU, G. and ZHANG, Z. (1998). Adaptive covariance estimation of locally stationary processes. Ann. Statist. $261-47$.

Munk, W., Worcester, P. and Wunsch, C. (1995). Ocean Acoustic Tomography. Cambridge Univ. Press. 
NEUMANN, M. H. and VON SACHS, R. (1997). Wavelet thresholding in anisotropic function classes and application to adaptive estimation of evolutionary spectra. Ann. Statist. 25 38-76.

YAGLom, A. M. (1987). Correlation Theory of Stationary and Related Random Functions 1, 2. Springer, New York.

Zurbenko, I. G. (1986). The Spectral Analysis of Time Series. North-Holland, New York.

DEPARTMENT OF STATISTICS

UNIVERSITY OF CALIFORNIA, RIVERSIDE

RIVERSIDE, CALIFORNIA 92521

E-MAIL:ks1@gauss.ucr.edu
DEPARTMENT OF MATHEMATICS

UNIVERSITY OF CALIFORNIA, SAN DIEGO

LA JOLLA, CALIFORNIA 92093

E-MAIL: mrosenblatt@ucsd.edu 


\section{CORRECTION}

SPECTRAL ANALYSIS FOR HARMONIZABLE PROCESSES

\section{By KeH-Shin LiI AND MurRay Rosenblatt}

The Annals of Statistics (2002) 30 258-297

In the statement of Theorem 5.3, page 273, $f_{a, b}\left(a^{\prime} \eta^{\prime}+\omega^{\prime}+2 \pi k\right)$ should be replaced by $f_{a, b}\left(\alpha^{\prime} \eta^{\prime}+\omega^{\prime}+2 \pi k\right)$ and $f_{a^{\prime}, b^{\prime}}\left(a^{\prime} \eta^{\prime}+\omega^{\prime}+2 \pi k^{\prime}\right)$ by $f_{a^{\prime}, b^{\prime}}\left(\alpha^{\prime} \eta^{\prime}+\right.$ $\left.\omega^{\prime}+2 \pi k^{\prime}\right)$. The theorem holds in the non-Gaussian case if

$$
\sup _{t} \sum_{\tau, t^{\prime}, \tau^{\prime}}\left|\operatorname{cum}\left(X_{t}, X_{\tau}, X_{t^{\prime}}, X_{\tau^{\prime}}\right)\right|<\infty .
$$

On the same page $f_{1,0}(\eta+\omega)$ should be replaced by $f_{1,0}(\alpha \eta+\omega) \ell(\alpha)$.

From page 292 on, wherever $a^{\prime} \eta^{\prime}+\omega^{\prime}$ occurs it should be replaced by $\alpha^{\prime} \eta^{\prime}+\omega^{\prime}$.

DEPARTMENT OF STATISTICS

UNIVERSITY OF CALIFORNIA, RIVERSIDE

RIVERSIDE, CALIFORNIA 92521

E-MAIL:ksl@gauss.ucr.edu
DEPARTMENT OF MATHEMATICS

UNIVERSITY OF CALIFORNIA, SAN DiEgo

LA JOLlA, CALIFORNIA 92093

E-MAIL: mrosenblatt@ucsd.edu

Received December 2002. 